\title{
Vinculin mediated axon growth requires interaction with actin but not talin
}

\author{
Pranay Mandal, Vivek Belapurkar, Deepak Nair, Narendrakumar Ramanan*
}

Centre for Neuroscience, Indian Institute of Science, Bangalore 560012, Karnataka, India

*Corresponding author

Narendrakumar Ramanan

Centre for Neuroscience

Indian Institute of Science

CV Raman Road, Malleswaram

Bengaluru 560012, India

Email: naren@iisc.ac.in

Tel: +91-80-2293 3532 


\title{
Vinculin-mediated axon growth requires interaction with actin but not talin
}

\begin{abstract}
Axon growth requires coordination of the actin cytoskeleton by actin-binding proteins in the extending neurites. Vinculin is a major constituent of focal adhesion but its role in neuronal migration and axon growth is poorly understood. We found that vinculin deletion in mouse neocortical neurons attenuated axon growth both in vitro and in vivo. Using different functional mutants of vinculin, we found that expression of a constitutively active vinculin significantly enhanced axon growth while the head-neck domain had a moderate inhibitory effect. Interesting, we found that vinculin-talin interaction was dispensable for axon growth and neuronal migration. Strikingly, expression of the tail domain delayed migration, increased branching and stunted axon. Inhibition of the Arp2/3 complex or abolishing the tail domain interaction with actin completely reversed the branching phenotype caused by tail domain expression without affecting axon length. Super-resolution microscopy showed increased mobile fraction of actin in tail domain expressing neurons. Our results provide novel insights into the role of vinculin and its functional domains in regulating neuronal migration and axon growth.
\end{abstract}

Keywords: Axon Growth/Neurite branching/Neuronal migration /Vinculin/Talin/

\section{Introduction}

Neuronal migration, axon growth and extension are intricately regulated processes that must be strictly coordinated for normal structural and functional development of the mammalian brain. These require coordination of the cellular cytoskeleton at the leading processes during neuronal migration and 
at the growth cone during axon growth (Dent et al, 2011; Lian \& Sheen, 2015). The growth cone at the axon tip is a highly dynamic structure that is in constant interaction with the environment. The growth cone movements are aided by the formation of actin-rich membranous structures called lamellipodia and filopodia (Luo, 2002). These structures are driven by constant polymerization and depolymerization of the actin cytoskeleton. The actin cytoskeletal dynamics are regulated by several actin-binding proteins (Ishikawa \& Kohama, 2007), which mediate polymerization, depolymerization and branching of actin filaments.

A critical first step in the growth and extension of neurites is filopodia formation. Filopodia are Factin rich structures that are essential for neurite formation and growth (Dent et al, 2007). Studies have shown that neurons that lack critical actin-binding proteins showed attenuated neuritogenesis and axon growth (Kwiatkowski et al, 2007). Extension of the cell membrane and growth of axon requires constant engagement of the actin cytoskeleton with the extracellular matrix (ECM) through integrin receptors on the cell surface (Letourneau et al, 1988). This engagement and the force required to push the membrane forward is generated by vinculin and talin (Mierke, 2009; Romero et al, 2020). Talin binds the integrin receptors and activates them and promotes their interaction with ECM (Calderwood et al, 2002; GarciaAlvarez et al, 2003). Vinculin is then recruited to focal adhesion sites and serves as a link between Talin/integrin receptors and the actin cytoskeleton (Atherton et al, 2015; Romero et al., 2020).

Vinculin is a $117 \mathrm{kDa}$ protein made up of three major domains - an N-terminal head (Vh), polyproline linker and a C-terminal tail domain (Vt) (Ziegler et al, 2006). Vinculin exists in an autoinhibited state in which all the ligand-binding sites are masked by intramolecular interactions between the 
head and tail domains (Bakolitsa et al, 2004; Borgon et al, 2004; Johnson \& Craig, 1994, 1995). Vinculin activation results in conformation changes that disassociates head-tail interaction exposing the binding sites (Bakolitsa et al., 2004; Borgon et al., 2004). Vinculin is found in neuronal growth cones and binds to talin and F-actin through its head and tail domains respectively and to several other actin-binding proteins (Burridge \& Mangeat, 1984; Letourneau \& Shattuck, 1989; Menkel et al, 1994; Ziegler et al., 2006). Vinculin has been shown to play a role in cell adhesion and motility in fibroblasts and PC12 cells. Vinculin null mice exhibit embryonic lethality at E10 with severe defects in heart and neural tube development (Xu et al, 1998a). Vinculin-deficient fibroblasts showed poor adhesion to substrate, less spreading, enhanced cell migration and cytoskeletal dynamics (Coll et al, 1995; Demali, 2004; Thievessen et al, 2013; Xu et al, 1998b). Vinculin has been shown to be important for filopodia and lamellipodia stability, neurite outgrowth and axon growth in vitro (Li et al, 2014; Sydor et al, 1996; Varnum-Finney \& Reichardt, 1994). Despite these studies, the requirement for vinculin and the role of different functional domains of vinculin for neuronal migration and axon growth in vivo is poorly understood.

In this study, we show that vinculin deletion in cortical neurons affects neuronal migration and attenuates axon growth both in vitro and in vivo. Furthermore, we found that different domains of vinculin affect axon growth to different extents. Expression of a constitutively active vinculin significantly enhanced axon growth while the tail domain alone attenuated axon growth. Vinculin tail domain expression caused an abnormal and enlarged cell soma, and highly branched neurites and stunted axon. Interestingly, abolishing vinculin-talin interaction did not affect neuronal migration and axon growth. The vinculin tail-induced branching phenotype was dependent on F-actin and Arp2/3 complex interactions. Thus, our findings provide novel insights into the role of vinculin in neuronal migration and axon growth. 


\section{Results and Discussion}

\section{Vinculin is necessary for axon growth}

To understand the requirement of vinculin for axon growth, we first ablated vinculin in neocortical neurons using CRISPR/Cas9-mediated gene deletion (Suppl. Fig. 1A, B). Neocortical neurons from postnatal day 1 (P1) mice were transfected either with empty Cas9n-T2A-GFP (Cas9-nickase) expressing plasmid or Cas9n-T2A-GFP plasmid along with a pair of guide RNAs (gRNAs) targeting exon 1 of $V c l$ gene. Neurons were fixed at 4 days post electroporation and plating and immunostained for $\beta$-III tubulin to label all neurites and GFP to identify transfected neurons. The control plasmid expressing neurons exhibited normal axon growth. In contrast, neurons expressing gRNAs targeting $V c l$ showed highly attenuated axon growth (Suppl. Fig. 1C, D). This was consistent with an earlier finding in which shRNAmediated knockdown of vinculin was shown to attenuate axon growth in cultured hippocampal neurons (Li et al., 2014). Next, we asked whether vinculin deletion also affected axon growth in vivo. For this, we introduced vinculin gRNAs into neural progenitor cells by in utero electroporation in embryonic day 14.5 (E14.5) embryos and analysed axon growth at E17.5. In the empty Cas9n-GFP plasmid (control) transfected embryos, the $\mathrm{GFP}^{+}$cells in the neocortex showed longer axons. However, the vinculin gRNA expressing neurons had much shorter axons (Suppl. Fig. 1E, F) suggesting that vinculin is necessary for axon growth. Vinculin null fibroblasts have been shown to exhibit increased cell mobility in cultures (Coll et al., 1995; Xu et al., 1998b). We therefore analysed neuronal migration at E17.5 following vinculin deletion in E14.5 embryos. In control plasmid electroporated embryos, we found $\mathrm{GFP}^{+}$cells present in all the layers of the neocortex (Suppl. Fig. 1G, H). In contrast, most of vinculin gRNA expressing neurons had migrated to the superficial layers indicating faster migration rate (Suppl. Fig 1G, H). Furthermore, we found that the vinculin gRNA expressing neurons exhibited shorter leading process compared to the 
control GFP ${ }^{+}$neurons (Suppl. Fig 1I, J). Together, these findings suggest that vinculin deletion attenuates neurite growth and enhances cell migration in vivo.

\section{Vinculin functional domains affect axon growth and cell migration differently}

Vinculin has three major domains - head, neck and tail domains, through which it interacts with other actin-binding proteins, kinases and F-actin. But the requirements for these domains for axon growth and neuronal migration is poorly understood. To study this, we expressed several N-terminal GFP-tagged vinculin mutants in cultured neocortical neurons and assessed for axon growth at 4 days in vitro (DIV) (Cohen et al, 2005; Cohen et al, 2006; Humphries et al, 2007) (Suppl. Fig. 2). These mutants included vinculin full length (Vcl-FL), vinculin N-terminal domain lacking the tail domain (Vcl-258, amino acids 1-258; Vcl-851, amino acids 1-851), vinculin tail domain (amino acids 881-1066) that binds actin (Vcl$\mathrm{T}$ ), a constitutively active form of vinculin (Vcl-T12) that has point mutations abolishing the head-tail interactions and a vinculin mutant that cannot bind PIP2 (Vcl-LD) (Chandrasekar et al, 2005; Cohen et al., 2005). Expression of each of these mutants affected axon growth to varying extents (Fig. 1A, B). VclFL expression did not increase axon growth any further compared to control plasmid transfected cells. However, Vcl-T12 expression significantly increased axon growth while the other mutants attenuated axon growth (Fig. 1B). We next asked whether Vcl-T12 also increased axon growth in vivo. For this, we electroporated embryos at E14.5 and analysed axon growth at P5. For comparison, we chose Vcl-T, which caused a severe attenuation of axon growth in cultured neurons. Similar to that seen in cultured cells, Vcl$\mathrm{T} 12$ was able to increase axon growth in vivo compared to control $\mathrm{GFP}^{+}$neurons. In contrast, $\mathrm{Vcl}-\mathrm{T}$ neurons exhibited highly stunted axon growth (Fig. 1C, D). Together these findings suggest that the full- 
length vinculin is required for normal axon growth while a constitutively active vinculin, that exists in an open conformation, can augment axon growth.

We next asked about the requirement for these domains for neuronal migration. During corticogenesis, neurons generated in the ventricular/subventricular zone migrate radially towards the pial surface and form distinct layers in an inside-out manner termed neocortical lamination (Nadarajah \& Parnavelas, 2002). We transfected the above vinculin mutants in E14.5 embryos and assessed cell migration three days later at E17.5. We did not see any defects in cell migration when Vcl-258 and Vcl851 were expressed as compared to control GFP transfection (Fig. 1E, F). However, when the constitutively active Vcl-T12 mutant was expressed, more $\mathrm{GFP}^{+}$cells were seen in layer 5/6 and less number in the ventricular zone/intermediate zone (VZ/IZ). But there was no change in the number of cells in the upper layers 2/3. In contrast, Vcl-T expressing cells showed delayed migration to the upper layers and an increase in the number of cells in the ventricular zone (Fig. 1E). Also, there were fewer cells in layers $2 / 3$. Together these findings indicated that lack of the vinculin tail domain (Vcl-258 and Vcl-851) does not affect neuronal migration while vinculin tail domain expression severely delayed cell migration from the ventricular zone/intermediate zone. Vcl-T12 appears to enhance migration from the intermediate zone compared to Vcl-T mutant, but not to the extent seen with vinculin deletion (Fig 1E, F). Given this interesting phenotype observed with Vcl-T12 and Vcl-T mutants, we next asked how these mutants affected migration at a later time point. For this, we electroporated embryos at E14.5 and analysed cell migration at P5. The Vcl-T12 expressing neurons that were seen enriched in layers 5/6 have reached their upper layer targets and there was no significant difference compared to control GFP expressing cells. In striking contrast, a greater number of Vcl-T expressing neurons were still seen in the lower layers (VZ/IZ 
and layers 5/6) (Fig. 2A, B). We further confirmed the defect in neuronal migration using immunostaining for Cux1, which is specifically expressed in layers 2-4 in the neocortex (Ferrere et al, 2006). We observed more $\mathrm{Cux}^{+}$cells in the lower layers in Vcl-T transfected embryos than those expressing control GFP (Suppl. Fig. 3). Interestingly, while analysing cell migration, we noticed that Vcl-T expressing neurons exhibited abnormal cell soma morphology. We analysed the area of neuronal soma and found that Vcl-T expressing cells had a much larger cell soma compared to control neurons (Fig. 2C, D).

\section{Talin binding is dispensable for vinculin-dependent axon growth}

Previous studies in fibroblasts have shown the interaction of vinculin with talin, a focal adhesion protein that binds to the integrin receptors, is important for mechanotransduction (Atherton et al, 2016). It has have shown that the amino acid alanine at position 50 in vinculin $\mathrm{N}$-terminal domain is critical for binding talin and that the alanine to isoleucine substitution $\left(\mathrm{Vcl}^{\mathrm{A} 50 \mathrm{I}}\right)$ abolishes this interaction (Bakolitsa et al., 2004; Cohen et al., 2006). We generated GFP-tagged full length mutant (GFP-Vcl-FL $\left.{ }^{\mathrm{A} 50 \mathrm{I}}\right)$, GFPVcl-851 ${ }^{\mathrm{A} 50 \mathrm{I}}$ and $\mathrm{Vcl}-258^{\mathrm{A} 50 \mathrm{I}}$ mutants and expressed it in cultured neocortical neurons. Interestingly, we found that the GFP-Vcl-FL ${ }^{\text {A50I }}$ did not affect axon growth when compared to Vcl-FL and empty vector (Fig. 2E, F). Similarly, the GFP-Vcl-851 A50I and Vcl-258 ${ }^{\mathrm{A} 50 \mathrm{I}}$ mutants did not exacerbate the attenuated axon growth phenotype caused by Vcl-851 and Vcl-258 mutants respectively (Fig. 2F). We next asked whether talin binding is necessary for cell migration. For this we compared cell migration at E17.5 following expression of $\mathrm{Vcl}-\mathrm{FL}^{\mathrm{A} 50 \mathrm{I}}$ and control GFP. We did not observe any difference in cell migration when Vcl-FL ${ }^{\text {A50I }}$ was expressed (Fig. 2G, H). Together our observations suggested that either talin-binding to vinculin is dispensable for axon growth and neuronal migration or that there are as yet unknown talinbinding residues in $\mathrm{N}$-terminus of vinculin. It is also likely that other actin-binding proteins such as $\alpha$ - 
actinin, which has been shown to bind vinculin and integrins compensates for lack of talin binding (Le et al, 2017; Roca-Cusachs et al, 2013).

\section{Vinculin tail domain induces excessive branching and enlarged cell soma}

Since Vcl-T affected neuronal morphology and migration, we sought to characterize further the functions of Vcl-T. We first looked at neurite branching and using Sholl analysis, we quantified the number of primary neurites arising from the cell soma and the number of secondary branches from the primary neurites. Compared to control neurons, we found that Vcl-T increased both the number of primary and secondary neurites (Fig. 3A, B). Neocortical neurons in culture have been shown to exhibit a stereotypic pattern of growth (Craig \& Banker, 1994). Following plating, first the neurons extend several filopodial and lamellipodial protrusions from the cell soma (stage 1) followed by extension of many neurites (stage 2). Next, one of these neurites grows rapidly to become the axon (stage 3) followed by dendritic growth (stage 4). We therefore asked how Vcl-T affected the early stages of neuronal growth. For this, primary neocortical neurons were dissociated, transfected with Vcl-T or control GFP and cultured in vitro. Cells were fixed at $24 \mathrm{~h}$ and $48 \mathrm{~h}$ and analysed for number of primary and secondary neurites, and the length of the longest neurite. At $24 \mathrm{~h}$ post-plating, the Vcl-T transfected neurons showed greater number of primary neurites that also showed more branches (secondary neurites) with no significant difference in the length of the longest neurite (Suppl. Fig. 4A, B). At $48 \mathrm{~h}$ post-plating, the number of primary and secondary neurites remained higher in Vcl-T transfected neurons compared to control neurons (Suppl. Fig. 4C, D). However, the length of the longest neurite was shorter in Vcl-T expressing neurons compared to control (Suppl. Fig. 4C, D). Since, a single longer neurite was not evident in Vcl-T expressing neurons, we wondered whether neuronal polarity was affected in these cells. We immunostained the 
cultures for the axon specific marker, Tau. We found that although shorter, the Vcl-T expressing neurons extended only one axon (Fig. 3A, B). Together, these findings suggested that although Vcl-T expression does not affect neuronal polarity, Vcl-T attenuated axon growth and increased neurite branching from an early stage of neurite growth.

We had earlier observed abnormal cell soma morphology of Vcl-T expressing neurons in vivo (Fig. 2C, D). We therefore asked whether this phenotype is also seen in cultured neurons. Neocortical neurons were transfected with Vcl-T or control GFP and grown in culture for 4 days. Cells were fixed and stained for rhodamine-conjugated phalloidin to label F-actin. We found that Vcl-T expressing neurons exhibited a larger cell soma indicating that Vcl-T expression increased cell spreading (Fig. 3C, D). This is in contrast to observations made in cultured fibroblasts, wherein Vcl-T expression has been shown to reduce focal adhesions resulting in a smaller cell area (Humphries et al., 2007; Mierke et al, 2008).

Vinculin is present in the leading edge of processes where it helps in binding to the substratum to promote migration (Bays \& DeMali, 2017). Since Vcl-T expressing neurons had shorter axons in vitro (Fig. 1A, B) and in vivo (Fig. 1C, D), we asked whether growth cone morphology is affected since improper growth cone can affect axon growth. For this, we analysed growth cone structure in the cultured neurons expressing Vcl-T and control GFP. We found that the area of the growth cone was smaller and appeared collapsed in Vcl-T compared to control GFP neurons (Fig. 3E, F). Together, these observations suggest that Vcl-T affects neuronal soma and growth cone morphology both in vitro and in vivo.

\section{Vcl-T-mediated branching phenotype requires Arp2/3 activity and actin-binding}


The attenuation of axon growth and the excessive branching phenotype caused by Vcl-T suggested that vinculin tail domain inhibits F-actin polymerization (Le Clainche et al, 2010) and this in turn stabilizes the F-actin filament, thereby allowing the binding of other proteins to induce branching. The actin cytoskeleton binds to a large array of actin-binding proteins, which regulate the structural changes in actin including polymerization and nucleation. The actin-related protein 2/3 (Arp2/3) complex is an important actin nucleator that binds to existing actin filaments and causes branching of actin filaments and neurites (Blanchoin et al, 2000; Mullins et al, 1998; Sturner et al, 2019). We hypothesized that Vcl-T-induced neurite branching is mediated by the Arp2/3 complex. For this, we transfected neocortical neurons with Vcl-T or control GFP and cultured them in the presence of $25 \mathrm{nM}$ of CK-666, a small molecule inhibitor of the Arp2/3 complex (Hetrick et al, 2013). Inhibition of Arp2/3 complex completely suppressed the branching phenotype and the number of primary and secondary neurites in Vcl-T expressing neurons was similar to that of control cells (Fig. 4A, B). However, inhibition of Arp2/3 complex failed to rescue the attenuated axon length caused by Vcl-T and caused further decrease in growth compared to Vcl-T expressing neurons (Fig. 4B).

The vinculin tail domain binds F-actin and crosslinks it into actin bundles (Huttelmaier et al, 1997; Menkel et al., 1994). Among several amino acid residues identified in Vcl-T domain that bind to actin, the isoleucine 997 to alanine mutant $\left(\mathrm{Vcl}_{-} \mathrm{T}^{\mathrm{I} 97 \mathrm{~A}}\right)$ was found to be the most impaired in actin binding (Thompson et al, 2014). We generated $\mathrm{Vcl}^{\mathrm{T}} \mathrm{T}^{\mathrm{I9} \mathrm{A}}$ to determine the effect of abolishing actin-Vcl-T interaction on axon growth and neuronal morphology. Neocortical neurons expressing Vcl-T ${ }^{1997 \mathrm{~A}}$ showed a decreased branching phenotype compared to Vcl-T expressing neurons and the number of primary and secondary neurites were comparable to that of control neurons (Fig. 4C, D). But similar to that seen with 
Arp2/3 inhibition, abolishing Vcl-T-actin interaction did not rescue axon growth and in fact, it exacerbated the stunted axon phenotype (Fig. 4D).

We next asked whether abolishing Vcl-T-actin interaction can rescue the enlarged cell soma phenotype. We found that $\mathrm{Vcl}-\mathrm{T}^{1997 \mathrm{~A}}$ mutant did not cause any increase in cell soma area as seen in VclT expressing neurons (Suppl. Fig. 5A, B). This suggested that Vcl-T expression affected actin dynamics resulting in an enlarged soma and abolishing this interaction with actin prevented enlargement of cell soma. We had earlier shown that Vcl-T caused a reduction in the area of the growth cone and this could be due to ability of Vcl-T to bind the barbed ends of F-actin preventing its polymerization (Fig. 3E, F) (Le Clainche et al., 2010). We therefore asked whether abolishing Vcl-T-actin binding can rescue the growth cone morphology and promote axon extension. For this, we transfected neurons with control GFP plasmid, Vcl-T or Vcl-T ${ }^{1997 A}$ along with LifeAct-mCherry to label actin. To assess growth morphology, Vcl-T ${ }^{1997 A}$ expressing neurons were imaged for LifeAct-mCherry expression. We found that the growth cone morphology was not rescued in the absence of actin binding suggesting that either Vcl-T binding recruits other actin-binding proteins that inhibit axon extension or that the head and neck domains are required for normal axon growth (Suppl. Fig. 5C, D).

Since abolishing actin-binding in $\mathrm{Vcl}-\mathrm{T}$ (Vcl-T ${ }^{1997 \mathrm{~A}}$ ) was able to decrease Vcl-T-induced branching, we were interested in knowing how the full-length vinculin that is deficient in actin binding would function in neurons. For this we generated a similar isoleucine 997 to alanine point mutation in full-length vinculin (GFP-Vcl-FL ${ }^{\text {I997A }}$ ) and expressed it in neurons. We found that Vcl-FL ${ }^{\text {1997A }}$ expression 
affected axon growth to a moderate but a significant extent. However, it did not induce or affect any branching (Suppl. Fig. 6).

\section{Abolishing actin binding rescues neuronal migration defects caused by Vcl-T}

We had seen that Vcl-T impaired neuronal migration in vivo (Fig. 1E, F). We next sought to determine whether abolishing actin interaction will rescue this defect in migration. For this, we electroporated Vcl-T, Vcl-T ${ }^{1997 A}$, control plasmid into E14.5 embryos and visualized neuronal migration three days later at E17.5. In contrast to the slower migration rate exhibited by Vcl-T expressing neurons, the $\mathrm{Vcl}-\mathrm{T}^{1997 \mathrm{~A}}$ neurons showed normal migration and were comparable to that seen in control embryos (Fig. 4E, F). We next looked at neuronal migration at a later time point (P5) following electroporation at E14.5. In control GFP transfection, the neurons had migration to the upper layers as expected while the Vcl-T expressing neurons were stalled in the lower layers (Fig. 4G, H). However, the Vcl-T ${ }^{1997 \mathrm{~A}}$ expressing neurons exhibited normal migration to the upper layers (Fig. 4G, H) indicating that abolishing actin binding rescued migration defect caused by Vcl-T. This was further confirmed by immunostaining for the upper layer marker, Cux1. In control GFP and Vcl- $\mathrm{T}^{\mathrm{I997A}}$ transfected embryos, all Cux $1^{+}$cells were seen in layers 2 to 4 suggesting normal migration. However, in Vcl-T transfected embryos, many Cux $1^{+}$ neurons were seen in the lower layers indicating retarded migration (Suppl. Fig. 7). Vcl-T expression was seen to impair axon growth in vivo (Fig. 1C, D). We asked whether Vcl-T $\mathrm{T}^{\mathrm{I} 997 \mathrm{~A}}$ mutant can rescue the axon growth defect caused by Vcl-T. For this, we analysed the length of the axon along the corpus callosum at P5. In control GFP transfected brains, the axons from the transfected hemisphere had crossed over to the contralateral hemisphere while in the Vcl-T transfected embryos, the axons were shorter and very few crossed the midline (Fig. 5A, B). However, in Vcl-T $\mathrm{T}^{\mathrm{I} 997 \mathrm{~A}}$ transfected brains, the axons were longer and 
crossed the midline but were significantly shorter compared to control neurons (Fig. 5A, B). Together, these observations suggested that abolishing actin binding was able to completely rescue the migration defect caused by Vcl-T but could only moderately rescue axon growth in vivo.

Vcl-T expression also resulted in an enlarged cell soma in vivo (Fig. 2C, D). We next asked whether inhibiting Vcl-T-actin binding can rescue the enlarged cell soma phenotype. For this, we measured the cell body area of $\mathrm{GFP}^{+}$neurons in the upper layers of the cortex at P5 in brain sections transfected with $\mathrm{Vcl}-\mathrm{T}^{1997 \mathrm{~A}}$ and compared the results with that of Vcl-T and control GFP expressing neurons. We found that the cell soma area of $\mathrm{Vcl}-\mathrm{T}^{1997 \mathrm{~A}}$ expressing neurons was comparable to that of control neurons and this was similar to that observed in in vitro culture experiments (Fig. 5C, D). Together these results suggested that the defects in migration and abnormal morphology of neurons caused by vinculin tail domain was due to abnormal interaction with actin and that abolishing this interaction could rescue both these defects in vivo and in vitro but not axon length.

\section{G-actin mobility is faster in neurons expressing vinculin tail domain.}

To understand the molecular mechanisms underlying the increased branching phenotype caused by $\mathrm{Vcl}-\mathrm{T}$, we studied actin mobility in cultured neurons. The Vcl-T ${ }^{1997 \mathrm{~A}}$ expression data indicated that actin-binding is necessary for the branching phenotype. Since Vcl-T has been shown to bind the barbed ends of F-actin and prevent its polymerization (Le Clainche et al., 2010), we hypothesized that this would increase the G-actin pool, thus making Arp2/3 complex to nucleate and generate branching. To test this hypothesis and to visualize actin in real-time, we co-electroporated neurons with LifeAct-mCherry along with control GFP or Vcl-T construct. LifeAct, the first 17 amino acids of actin-binding protein 140 from 
S. cerevisiae, binds to actin filaments without modifying its function (Riedl et al, 2008). mCherry was tagged to LifeAct for visualization of actin in cells. To study the behaviour of actin in neurons, we photobleached mCherry in a small region close to the edge of the longest neurite by using a high-power laser of $561 \mathrm{~nm}$ wavelength and monitored fluorescence recovery over time (FRAP). The fluorescence recovery was fit as a single exponential function of time and half-time of recovery and mobile fraction was calculated from the curve fitting (Fig. 5E-G). In Vcl-T expressing neurons there was recovery of a higher mobile fraction of LifeAct $(0.7655+/-0.06361)$ when compared to mobile fraction of control cells $(0.3071+/-0.03738)($ Fig. 5F). We also observed a significant increase in t-half of recovery in presence of Vcl-T (3227ms +/- 647.2ms) compared to control (941.5ms +/- 171.0ms) (Fig. 5E). This increase in mobile fraction could be due to either an increase in G-actin pool or impaired F-actin dynamics (actin treadmilling). Interestingly, neurons expressing the $\mathrm{Vcl}-\mathrm{T}^{\mathrm{I} 997 \mathrm{~A}}$ mutant, that is defective in actin binding, showed a complete rescue in the mobile fraction $(0.3268+/-0.03837)$ and t-half $(1139 \mathrm{~ms}+/-206.2 \mathrm{~ms})$ of $\operatorname{actin}($ Fig. 5F, G).

To further characterize the effect of Vcl-T on actin mobility, we expressed Vcl-T along with mEOS3.2-LifeAct (Zhang et al, 2012). Using photoactivation properties of mEOS3.2, we performed single particle tracking based photoactivation localization microscopy on LifeAct in transfected cells. High-resolution intensity map and single particle trajectories were generated for each neuron (Fig. 5H). Instantaneous diffusion coefficient (D) and mean squared displacement (MSD) were calculated and used to estimate diffusion dynamics and confinement of the LifeAct molecules. The trajectories displayed a wide range of diffusion coefficient and a significant difference in distribution of logarithmic values of diffusion coefficient was observed, indicating that mEOS3.2-LifeAct was more mobile in presence of Vcl- 
T (Fig. 5I). In presence of Vcl-T, instantaneous mEOS3.2-LifeAct showed increase in diffusion coefficient $(0.2570 \mu \mathrm{m} 2 / \mathrm{sec}$, IQR: $0.1012-0.5867 \mu \mathrm{m} 2 / \mathrm{sec})$ when compared to control $(0.1749 \mu \mathrm{m} 2 / \mathrm{sec}, \mathrm{IQR}$ : $0.06114-0.4980 \mu \mathrm{m} 2 / \mathrm{sec}$ ) after pooling all the trajectories obtained (Fig. $5 \mathrm{~K}$ ). To rule out the possibility of cell to cell variation in diffusion coefficient, we compared the median of diffusion coefficient from all cells extracted individually and found the difference to be significant (Fig. 5L). These values were consistent with the pooled analysis. The average MSD of LifeAct saturated with time in both conditions indicating confined diffusion in both cases. However, the saturation values of LifeAct in the presence of Vcl-T were higher than the control indicating lesser confinement compared to control (Fig. 5J). This was also validated by significant difference in the area under the curve for MSD, for the average MSD curves generated for each cell (Fig. 5M). Together these observations confirm that the actin molecules became less confined and more mobile in the presence of vinculin tail domain. This could facilitate nucleation of actin and branching by the Arp2/3 complex resulting in enhanced branching induced by the Vcl tail domain.

Here, we demonstrate that vinculin plays a critical role in neuronal migration in vivo and in axon growth in vitro and in vivo. Although full length vinculin is required for optimal axon growth, the mutants used in this study provide novel insights into some of the functional domains of vinculin. The constitutively active vinculin greatly enhanced axon growth both in vitro and in vivo. This observation opens up the question of whether vinculin activation can promote axon growth on inhibitory substrates and also raises the possibility that inhibitory substrates could likely affect axon growth by blocking vinculin-dependent actin dynamics. We also observed that preventing vinculin-talin binding did not affect migration and axon growth indicating that this interaction is dispensable for these vinculin-dependent functions, in contrast to that seen in fibroblasts. Interestingly, vinculin tail domain affected neuronal 
morphology in several ways compared to the other functional domain mutants of vinculin. Among these was a striking increase in neurite branching and enlarged cell soma, with the latter phenotype being different than that seen with fibroblasts, in which Vcl-T expression resulted in a smaller cell. These phenotypes could be rescued by abolishing interaction of the tail domain with actin and by inhibiting the Arp2/3 complex. Vcl-T, by binding to the barbed end of F-actin prevents polymerization and thus resulting in an increase in mobile actin pool. This in turn stabilizes F-actin and causes an Arp2/3-dependent branching and the process continues, resulting in highly branched but shorter neurites. It will be interesting to see how these Vcl-T will affect the localization and properties of the other actin-binding proteins that play a role in axon growth. 


\section{Materials and methods}

Animals. For all experiments, C57B1/6J mice were used. All experiments were conducted in accordance with the animal care standards of the Institutional Animal Ethics Committee of the authors' institution.

Constructs. Sequences of guide RNAs (gRNAs) for deleting Vcl gene was selected using DeskGen software (www.deskgen.com) and two gRNAs that bind to flanking regions of translational start site were chosen. The chosen gRNA sequences are as follows: Vcl-gRNA-1, 5'- GCGTACGATCGAGAGCATCC3' and Vcl-gRNA-2, 5'-GCTAGCGGGGCGGCGTACCG-3'. The gRNAs were first individually cloned into pX330-U6-BB-CBh-hSpCas9 plasmid (Addgene No: 42230) using BbsI site. These two gRNAs were then subcloned into pSpCas9n(BB)-2A-GFP plasmid (pX461; Addgene No: 48140). We made use of Cas9 nickase (Cas9n) to decrease any potential OFF-target effects. The resultant vector was named pX461-Vclsg1+2--Cas9n-2A-GFP. pSpCas9n(BB)-2A-GFP (PX461) and pX330-U6-Chimeric_BB-CBh-hSpCas9 were a gift from Feng Zhang (Addgene plasmid \# 48140, 42230; http://n2t.net/addgene:48140; RRIDs: Addgene_48140, Addgene_42230).

The mouse $V c l$-encoding gene (Vcl-FL) was amplified from cDNA synthesized from whole brain mRNA. Vcl-FL ${ }^{\mathrm{A} 50 \mathrm{I}}$ was generated from Vcl-FL by mutating the alanine residue at 50th position to isoleucine using site-directed mutagenesis (SDM). Vcl-FL ${ }^{1997 \mathrm{~A}}$ was generated from Vcl-FL by mutating isoleucine residue at 997 th position to alanine using SDM. Vcl-LD was generated from Vcl-FL by mutating 1060th residue from arginine to glutamine and residue at 1061st from lysine to glutamine by using SDM. Vcl-258 was amplified from full length vinculin by using the following primers: Forward 5'-CTTGAATtCGATGCCGGTGTTTCACACG-3' $\quad$ Reverse 5'CGGGGTACCCCAGGCGTCTTCATCCC-3'. Vcl-258 ${ }^{\text {A50I }}$ was generated from Vcl-258 by mutating alanine residue at 50th position to isoleucine using SDM. Vcl-851 was amplified from full length vinculin 
using following primer: Forward, 5'-CTTGAATTCGATGCCGGTGTTTCACACG-3' and Reverse, 5'CGCGGTACCCTCTTCTGGTGGTGGGG-3'. Vcl-851 ${ }^{\text {A50I }}$ was generated from Vcl-851 by mutating alanine residue at 50th position to isoleucine using SDM. Vcl-T12 was generated by mutating residues 974th from glutamate to alanine, 975th from lysine to alanine, 976th from arginine to alanine and 978th from arginine to alanine from full length vinculin by using SDM. Vcl-T was amplified from Vcl-FL by using these primers: Forward 5'-CTTGAATTCGAAGGATGAAGAGTTCCCTG-3' and Reverse 5'CGCGGATCCCTGGTACCAGGGAGTCTTTC-3'. All these clones were generated in pCAG-C1 vector. pCAG-C1 was generated from pEGFP-C1 vector by replacing the CMV promoter by CAG promoter from pCAGEN, a gift from Connie Cepko (Addgene plasmid \# 11160; http://n2t.net/addgene:11160; RRID: Addgene_11160). Vcl-T ${ }^{1997 A}$ clone was generated from Vcl-T by site-directed mutagenesis of the 997th amino acid residue from isoleucine to alanine. LifeAct-mCherry was a gift from Dr. Paul Bridgman, Washington University School of Medicine, St. Louis, MO. mEos3.2-C1 was a gift from Michael Davidson \& Tao Xu (Addgene plasmid \# 54550; http://n2t.net/addgene:54550; RRID: Addgene_54550). Vcl-T-mEOS3.2-LifeAct was generated by sub-cloning a Vcl-T-T2A fragment in mEOS3.2-LifeAct clone.

Site-directed mutagenesis. Complementary primers of 30-40 bp were designed with the mutation site in the middle of the sequence and $50 \mathrm{ng}$ of template DNA was used along with 25 pmoles each of the mutant primers. 2 min extension time for every $1 \mathrm{~kb}$ of template length was used. PCR was for a total of 18 cycles and the following cycling conditions were used: $95^{\circ} \mathrm{C}$ for $3 \mathrm{~min} ; 68^{\circ} \mathrm{C}$ for $(2 \mathrm{~min} / \mathrm{kb}) ; 18$ cycles; $72^{\circ} \mathrm{C}$ for $10 \mathrm{~min}$; end at $4^{\circ} \mathrm{C}$. The reaction mixture was digested with DpnI enzyme to remove template DNA and ligation was set with T4DNA ligase for $1 \mathrm{~h}$ at room temperature. A small aliquot of the ligation reaction 
was transformed into DH10B competent cells and transformants were selected on LB+antibiotic plates. The site-directed mutation was confirmed by DNA sequencing of both strands of at least 2 independent clones.

Immunocytochemistry. Neurons were fixed using 4\% PFA + 4\% sucrose in PBS for 20 min and washed in PBS, 2x10 min. The cells were permeabilized with $0.1 \%$ Triton-X100 in PBS for 5 min and blocked with $10 \%$ BSA in PBS for 30 min at room temperature with gentle rocking. Primary antibody incubation was done in $3 \% \mathrm{BSA}$ for 1 hour at $4^{\circ} \mathrm{C}$ with gentle rocking and then washed with PBS, 3x 2 min. Secondary antibody incubation was done in $3 \%$ BSA for $30 \mathrm{~min}$ at room temperature, washed with PBS, 3x2min and mounted with VECTASHIELD mounting medium with DAPI (\#H-1000, Vector Laboratories, USA). The following primary antibodies were used: anti-GFP (\#GFP-1020, 1:1000, Aves labs, Inc. USA), anti- $\beta$-III tubulin (\#Tuj, 1:1000, Aves labs, Inc. USA), anti-Tau (\#314002, 1:1000, Synaptic Systems, Germany). The following secondary antibodies from Invitrogen Inc., were used: AlexaFluor (AF)-488 conjugated anti-chicken (\# A11039, 1:1000), AF-594 conjugated anti-mouse (\#A21203, 1:1000), AF-594 conjugated anti-rabbit (\# A11012, 1:1000).

Phalloidin staining. Rhodamine-phalloidin (\#6876, 1:125, Setareh Biotech, USA) was added along with secondary antibody during immunocytochemistry.

Immunohistochemistry. Immunohistochemistry was done as follows: embryonic or post-natal brains were fixed by transcardial perfusion using ice-cold PBS followed by $4 \%$ PFA, post-fixed in $4 \%$ PFA overnight and then cryopreserved in $30 \%$ sucrose until the brains were saturated with sucrose and sunk to 
the bottom of the tube, and frozen at $-80^{\circ} \mathrm{C}$ until use. The frozen brains were sectioned at $20 \mu \mathrm{m}$ thickness, collected on Superfrost plus slides (Brain Research Laboratories, USA) and dried overnight before staining. Blocking/permeabilization was done in blocking solution (3\% bovine serum albumin $+0.3 \%$ Triton-X100 + 1\% goat serum in PBS) for $1 \mathrm{~h}$ at RT. Primary antibody incubation was done in the blocking solution overnight at $4{ }^{\circ} \mathrm{C}$ with gentle rocking and washed with PBS for $5 \times 5 \mathrm{~min}$. Secondary antibody was incubated in the blocking solution for $1 \mathrm{~h}$ at RT, washed with PBS for $5 \times 5$ min and slides were mounted with VECTASHIELD mounting medium with DAPI (Vector Laboratories, USA). The following primary antibodies were used: anti-GFP (GFP-1020, 1:1000, Aves labs, Inc. USA), anti-Cux1 (\#sc-13024, 1:50, Santa Cruz Biotechnology, USA). The following secondary antibodies from were used: AlexaFluor-488 conjugated anti-chicken (\#A11039, 1:1000,), AlexaFluor-594 conjugated anti-rabbit (\#A11012, 1:1000).

Neuronal culture and transfection. Neocortex from C57B1/6 pups (postnatal day 1-3) was dissected in Hibernate-A medium (\#A1247501, Gibco, USA), digested with papain (\#LS003124, Worthington, USA) for $30 \mathrm{~min}$ at $30^{\circ} \mathrm{C}$ and dissociated with micropipette tips in Neurobasal-A medium (\#10888022) supplemented with glutaMAX (\#35050061, Gibco, USA), B27 (\#17504044, Gibco, USA) and 33 U/ml penicillin/streptomycin. The tissue was gently titrated, cells were pelleted by centrifugation, and washed twice with Hibernate-A medium. Cells were then plated at a density of 125 cells $/ \mathrm{mm}^{2}$ on coverglasses (\#41001112, Glaswarenfabrik Karl Hecht, Germany) coated with poly-L-lysine (\# P2636, Sigma, USA) and laminin (\#354232, BD, USA). Poly-L-lysine $(50 \mu \mathrm{g} / \mathrm{ml})$ coating was done for $2 \mathrm{~h}$ at $37^{\circ} \mathrm{C}$, washed thrice with Milli-Q water and dried at RT for $15 \mathrm{~min}$ followed by laminin $(10 \mu \mathrm{g} / \mathrm{ml})$ coating for $2 \mathrm{~h}$ at 37 ${ }^{\circ} \mathrm{C}$. Cultures were incubated at $37^{\circ} \mathrm{C}$ with $5 \% \mathrm{CO} 2$. After allowing for cells to attach to poly-L-lysine coverglass (20 min post-plating), the medium was gently exchanged with new neuronal culturing medium. 
After 4 days in culture, neurons were fixed in $4 \%$ PFA+4\% sucrose in PBS for 20 min. Neuronal transfection was done just before plating using Nucleofection (Lonza, Switzerland) according to the manufacturer's instructions.

Drug treatment. For inhibiting Arp2/3 protein complex in vitro, the pharmacological inhibitor CK-666 (Sigma Aldrich, SML0006) was used at a concentration of 25nM. CK-666 or DMSO (control) was added at $24 \mathrm{~h}$ after plating the neurons and neurons were allowed to grow for more 3 days.

In utero electroporation. In utero electroporation for different mutants constructs of $V c l$ were done on embryonic day 14.5 (E14.5) in C57bl/6J embryos by injecting 1-2 $\mu \mathrm{l}$ of $1 \mu \mathrm{g} / \mu \mathrm{l}$ DNA in the lateral ventricle of one of the cerebral hemispheres. Electroporation was done using ECM 830 square wave electroporation system (BTX, USA) using $5 \mathrm{~mm}$ tweezertrode. The following parameters were used: voltage, 50V; pulse length, 50 milliseconds; number of pulses, 5 pulses; pulse interval, 100 milliseconds. Brains were harvested at either embryonic day 17.5 (E17.5) or at post-natal day 5 (P5) for immunostaining.

Fluorescence Recovery After Photobleaching (FRAP). FRAP was performed on an inverted fluorescence motorized fluorescence microscope (IX83 Olympus, Japan) equipped with a 100X 1.49 NA PL-APO objective and maintained at $37^{\circ} \mathrm{C}$ with the aid of a whole microscope temperature-controlled chamber. Neuronal primary cultures were loaded in open chamber (Ludin chamber, Life Imaging Services, Switzerland). The image acquisition was controlled by Metamorph (Molecular devices). Image acquisition was performed by illuminating with 488 or $561 \mathrm{~nm}$ laser and fluorescence were collected with filters to observes Green and Red fluorescence proteins in total internal reflection microscopy mode at 
$50 \mathrm{~Hz}$ (Gataca Systems, France). Photo bleaching was done by using 561nm laser line for $60 \mathrm{~ms}$ in a circular region of diameter 20 pixels close to the end of the longest neurite. For baseline normalization, 10 images were acquired before photobleaching. To observe the recovery of fluorescence 400 frames were acquired after photobleaching sequence. For comparison between different experiments average of prebleached values were normalized to zero. Percentage calculation and normalization were performed using Microsoft Excel software. The recovery of fluorescence was fit to One-Phase association equation in GraphPad Prism. The quantification of recovery of fluorescence, mobile fraction and T-half graphs were generated from the curve fitting.

Single Particle Tracking (SPT). Single particle tracking using photoactivation was performed on live neurons expressing mEOS3.2 as previously described (Nair et al., 2013). These cells were loaded in an open chamber and photoactivated using a 405nm laser (Omicron, Gataca Systems, France) and the images were acquired using 561nm laser (Cobolt, Gataca Systems, France). The power of lasers was adjusted to keep the number of the stochastically activated molecules constant and well separated during the acquisition. Continuous acquisition of 20000 frames of images at $50 \mathrm{~Hz}(20 \mathrm{~ms})$ were captured using a sensitive EMCCD camera (Evolve, Photometric). Image acquisition was controlled using MetaMorph software (Molecular Devices). Super resolved intensity images and single particle trajectories were obtained using custom built analysis for high density single particle tracking which were described previously (Nair et al, 2013).

Data analyses and quantifications. Western blot quantification was done by using ImageJ. Longest neurite length was measured using the 'traced line' tool in MetaMorph software (Molecular Devices). 
Numbers of primary and secondary neurites in cultured neurons were counted using Sholl analysis for different vinculin mutants. Cell body area was measured using 'trace area' tool in MetaMorph software (Molecular Devices). Numbers of cells in different layers of cortex was counted in ImageJ by using 'cell counter' plugin. All the graphs were made by using GraphPad Prism software.

Statistical analyses. One-way ANOVA followed by Tukey's post hoc test or two-tailed Student's $t$ test was used to determine statistical differences between groups using GraphPad Prism software. Error bars in figures are mean \pm SEM unless otherwise stated. For statistical difference between frequency distribution of logarithmic values of diffusion coefficients we used Kolmogorov-Smirnov test. Diffusion coefficients were compared by unpaired $t$ test. Wilcoxon matched-pair signed rank test was used to conclude the difference between MSD curves. Area under curve was calculated and compared using Mann-Whitney test.

Image acquisition. Fluorescent images were acquired using Nikon Eclipse 80i upright fluorescent microscope (Nikon Instruments, USA), and confocal LSM880 microscope (Zeiss, Germany). Images were acquired and pseudo coloured using MetaMorph software (Molecular Devices) for Nikon Eclipse 80i microscope and Zen software for confocal LSM880 microscope. 


\section{LEGENDS}

Figure 1. Different requirement of functional domains of vinculin for axon growth and cell migration in vivo and in vitro. (A) Primary neocortical neurons from P0.5 mouse pups were electroporated with different N-terminal GFP-tagged domain constructs of Vinculin and were grown for 4 DIV and immunostained for GFP. Absence of either head and neck (Vcl-T), neck and tail (Vcl-258) or tail alone (Vcl-851) and PIP2-binding defective mutant (Vcl-LD) attenuated axonal growth, while the constitutively active vinculin (Vcl-T12) enhanced axon growth while the full-length (Vcl-FL) did not have any effect. (B) Quantification of the length of longest neurite of neurons expressing different mutants of vinculin shown in (A) ( $\mathrm{n}=250-300$ cells). (C) Neocortical progenitors in E14.5 embryos were transfected with the domain deleted constructs of vinculin by in utero electroporation (IUE). The pups were sacrificed at P5 and axons were visualized using anti-GFP immunostaining (green). The Vcl-T transfected neurons exhibited highly stunted axons compared to the control GFP neurons. In contrast, Vcl-T12 expression increased axon growth significantly. Arrows indicate the axon terminal along the corpus callosum. Dashed lines represent the longitudinal fissure separating the two cerebral hemispheres. (D) Quantification of axon length from (C) (n=3 animals). (E) IUE was performed at E14.5 and the embryos were harvested at E17.5. $20 \mu \mathrm{m}$ brain sections were prepared and immunostained for GFP to study cell migration. The number of $\mathrm{GFP}^{+}$neurons in different cortical layers were counted. LV, lateral ventricle; IZ/VZ, intermediate zone/ventricular zone; V-VI, layers 5/6; II-IV, layers 2-4. (F) Quantification of the percentage of GFP+ neurons in different layers of cortex from (E). More Vcl-T expressing neurons were seen in the lower layers compared to control indicating delayed migration whereas $\mathrm{Vcl}-\mathrm{T} 12^{+}$neurons showed faster migration compared to control. ( ${ }^{*} p=0.0254$, Ctrl-Vcl-T12, ${ }^{*} p=0.0258$, Ctrl-Vcl-T; $\mathrm{n}=3$ animals). Scale, 
$100 \mu \mathrm{m}(\mathrm{A}), 2 \mathrm{~mm}(\mathrm{C}), 400 \mu \mathrm{m}(\mathrm{E}) . * * p<0.01, * * * p<0.001, * * * * p<0.0001$. ns, non-significant. One-way ANOVA and Tukey's post-hoc test in (B) and (F). Two-tailed student's $t$ test in (D).

\section{Figure 2. Interaction of talin with vinculin is dispensable for axonal growth and neuronal migration.}

(A) Neocortical progenitors in E14.5 embryos were electroporated by IUE with control GFP, GFP- VclT12 or GFP-Vcl-T and pups were analysed at P5 for neuronal migration. Vcl-T expressing neurons were still in migratory phase even at P5 while Vcl-T12 expressing cells had migrated to upper layers of cortex. (B) Quantification of the percentage of $\mathrm{GFP}^{+}$neurons in different layers of cortex from (A) (n=3 animals). (C) Representative images of neurons transfected with either empty GFP (control) or Vcl-T in vivo in E14.5 embryos. The Vcl-T expressing neurons exhibited larger and abnormal cell soma compared to control neurons. (D) Quantification of cell soma area from (C) ( $n=100$ cells, $n=3$ animals). (E) Primary mouse neocortical neurons were electroporated with GFP-tagged vinculin constructs that contained the head domain but were defective in talin binding ( Vcl-FL ${ }^{\mathrm{A} 50 \mathrm{I}}, \mathrm{Vcl}-258^{\mathrm{A} 50 \mathrm{I}}$ and $\left.\mathrm{Vcl}-851^{\mathrm{A} 50 \mathrm{I}}\right)$. Length of the longest neurite was compared with the corresponding mutant having intact talin binding site (Vcl-FL, Vcl258 and Vcl-851). (F) Quantification of longest neurite length from (E). Abolishing talin-vinculin interaction does not affect axonal growth in vitro. ( $\mathrm{n}=3$ animals). (G) IUE was performed using control GFP and Vcl-FL ${ }^{\text {A50I }}$ plasmids and the embryos were harvested at E17.5 to study migration. Inhibition of talin-vinculin interaction did not affect neuronal migration. (H) Quantification of the percentage of GFPpositive neurons in different layers of cortex $(\mathrm{G}) .(\mathrm{n}=3$ animals) ( $\mathrm{LV}$, lateral ventricle; IZ/VZ, intermediate

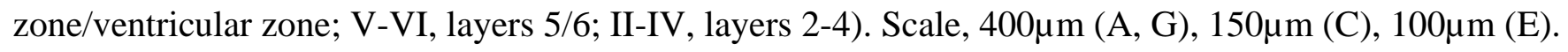
$* * * * p<0.0001$; ns, non-significant. One-way ANOVA and Tukey's post-hoc test (B, F and H). Two-tailed student's $t$ test (D). 


\section{Figure 3. Vinculin tail domain increases neurite branching, cell soma area, and affects growth cone}

morphology in vitro. (A) Neocortical neurons from P0.5 pups were electroporated with Vcl-T and control GFP plasmid and grown for 4 DIV. Cells were fixed and immunostained for GFP (to label transfected neurons) and Tau (to label axons). Vcl-T expression increased both the number of primary and secondary neurites but did not affect polarity as seen by only one $\mathrm{Tau}^{+}$neurite. Non-transfected neurons (white arrows) and dead cells (yellow arrows). (B) Quantification of number of Tau-positive neurites, and number of primary and secondary neurites from (A) ( $n=100$ cells). (C) Representative images of neurons expressing either empty GFP (control) or Vcl-T plasmid at 4DIV. Neurons were immunostained for GFP and rhodamine-conjugated phalloidin (Rho-Pha) to visualize cell soma in vitro. Vcl-T expressing neurons had a wider spread of cell soma compared to control. (D) Quantification of the cell soma area in control and Vcl-T expressing neurons from (C) (n=100 cells). (E) Representative images from live imaging of control and Vcl-T expressing neurons co-transfected with LifeAct-mCherry to label actin. Vcl-T expression caused a smaller or collapsed growth cone morphology. (F) Quantification of the growth cone

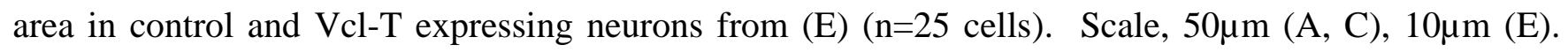
$* * * p<0.001, * * * * p<0.0001 ;$ ns, non-significant. Two-tailed student's $t$ test.

Figure 4. Blocking Arp2/3 complex and abolishing actin-Vcl-T interaction decreases branching but does not rescue axon length. (A) Representative images of neurons transfected with Vcl-T and treated either with DMSO (vehicle) or with 25nM CK-666 (Arp2/3 complex inhibitor) dissolved in DMSO. VclT neurons exhibited enhanced neurite branching and this abolished in the presence of CK-666. (B) Quantification of longest neurite length, number of primary and secondary neurites of neurons from (A) 
( $\mathrm{n}=100$ cells) (C) Neurons transfected with control, Vcl-T and Vcl-T defective in actin-binding (Vcl$\mathrm{T}^{\mathrm{T} 997 \mathrm{~A}}$ ) constructs and immunostained for GFP after 4DIV. Abolishing interaction of Vcl-T with actin reduced neurite branching to that seen in control cells. (D) Quantification of longest neurite length, number of primary and secondary neurites of neurons from (C) (n=300 cells). (E) Representative images of brain sections from E17.5 embryos following electroporation at E14.5 with control, Vcl-T and Vcl-T ${ }^{1997 \mathrm{~A}}$ constructs. Abolishing actin-Vcl-T interaction rescues neuronal migration defect caused by Vcl-T. (F) Quantification of the percentage of $\mathrm{GFP}^{+}$neurons in different layers of cortex from (E) (n=3 animals). (G) Analysis at post-natal day 5 (P5) also showed almost complete rescue of migration when actin-Vcl-T interaction is abolished. (H) Quantification of the percentage of $\mathrm{GFP}^{+}$neurons in different layers of cortex and in IZ/VZ from $(\mathrm{G})\left(\mathrm{n}=3\right.$ animals). Scale, $50 \mu \mathrm{m}(\mathrm{A}, \mathrm{C}), 400 \mu \mathrm{m}(\mathrm{E}, \mathrm{G}) .{ }^{*} p<0.01, * * * p<0.001$, ****p<0.0001; ns, non-significant. Two-tailed student's $t$ test in (B, D). One-way ANOVA and Tukey's post-hoc test in $(\mathrm{F}, \mathrm{H})$.

Figure 5. Abolishing actin-Vcl-T interaction reduces cell soma area in vivo and Vcl-T increases actin mobility in vitro. (A) Representative images of brain sections electroporated with control, Vcl-T and Vcl$\mathrm{T}^{1997 \mathrm{~A}}$ constructs. Vcl- $\mathrm{T}^{1997 \mathrm{~A}}$ defective in actin-binding only partially rescues axon growth deficit caused by Vcl-T. Arrows indicate the axon terminals along the corpus callosum. Dashed lines represent the longitudinal fissure separating the two cerebral hemispheres. Scale, 2mm. (B) Quantification of length of axons from (A) (n=3 animals. *p=0.0193, Vcl-T-Vcl-T ${ }^{1997 A} ; * p=0.0170, \quad$ Ctrl-Vcl-T ${ }^{1997 A}$.

Representative images of brain sections showing cell soma morphology of neurons expressing control GFP, Vcl-T and Vcl-T ${ }^{1997 A}$. Vcl-T expressing neurons exhibited abnormal soma (arrows) compared to control neurons, and this was rescued when actin-Vcl-T interaction was abolished. Scale, 150 $\mu$ m. (D) 
Quantification of neuronal cell soma area from $(\mathrm{C})(\mathrm{n}=100$ cells, $\mathrm{n}=3$ animals $) .{ }^{*} * * *<0.0001$; ns, nonsignificant. (E-G) Neurons were transfected with control GFP, Vcl-T or Vcl-T ${ }^{1997 A}$ along with LifeActmCherry to visualize actin. Fluorescence recovery curve $(\mathbf{E})$, mobile fractions $(\mathbf{F})$ and half-time $(\mathbf{G})$ of recovery of fluorescence of LifeAct-mCherry after FRAP of neurons transfected with control, Vcl-T and Vcl-T $\mathrm{T}^{1997 \mathrm{~A}}$ constructs, showing that neurons expressing Vcl-T have a higher mobile fraction and higher thalf of actin compared to control neurons. Abolishing actin-Vcl-T interaction $\left(\mathrm{Vcl}-\mathrm{T}^{\mathrm{I} 997 \mathrm{~A}}\right)$ reduces the mobile fraction and reduces t-half to normal level. ( $\mathrm{n}=10$ cells). ${ }^{* *} p<0.01$, $* * * * p<0.0001$; ns, nonsignificant. (H-M) Single particle tracking of mEOS3.2-LifeAct in control and Vcl-T expressing neurons. (H) Representative super-resolution intensity map reconstructed from single molecule localization (left) and single particle trajectories (right) generated from neurons expressing mEOS3.2-LifeAct. White arrows indicate the extensions of the neurite. Scale, $10 \mu \mathrm{m}$. The regions marked by arrows are represented below. For each region indicated by arrow, the magnified super-resolution intensity map (SR) and single particle trajectories (SPT) for the same region is shown. Scale, $2 \mu \mathrm{m}$. (I) Normalized cumulative distribution of logarithmic values of instantaneous diffusion coefficient of the generated tracks (Mean +/-SEM). ****p $p<0.0001$ (Kolmogorv-Smirnov test). (J) Plot of average mean squared displacement (MSD) of all trajectories above 6 frames for control and Vcl-T. ****p<0.0001 (Wilcoxon matched-pairs signed rank test). (K) Pooled values of instantaneous diffusion coefficient of all the detected trajectories from control and Vcl-T (median, IQR). ${ }^{* * *} p=0.0002$ (Unpaired $\mathrm{t}$ test). (L) Distribution of median diffusion coefficients of each neuron compared across the conditions. ${ }^{* *} p=0.0039$ (Unpaired t test). (M) Distribution of area under curve for average MSD for trajectories obtained from each cell of control versus Vcl-T. * $p=0.0469$ (Mann-Whitney test). 


\section{References}

Atherton P, Stutchbury B, Jethwa D, Ballestrem C (2016) Mechanosensitive components of integrin adhesions: Role of vinculin. Exp Cell Res 343: 21-27

Atherton P, Stutchbury B, Wang DY, Jethwa D, Tsang R, Meiler-Rodriguez E, Wang P, Bate N, Zent R, Barsukov IL et al (2015) Vinculin controls talin engagement with the actomyosin machinery. Nature communications 6: 10038

Bakolitsa C, Cohen DM, Bankston LA, Bobkov AA, Cadwell GW, Jennings L, Critchley DR, Craig SW, Liddington RC (2004) Structural basis for vinculin activation at sites of cell adhesion. Nature 430: 583586

Bays JL, DeMali KA (2017) Vinculin in cell-cell and cell-matrix adhesions. Cellular and molecular life sciences : CMLS 74: 2999-3009

Blanchoin L, Pollard TD, Mullins RD (2000) Interactions of ADF/cofilin, Arp2/3 complex, capping protein and profilin in remodeling of branched actin filament networks. Curr Biol 10: 1273-1282

Borgon RA, Vonrhein C, Bricogne G, Bois PR, Izard T (2004) Crystal structure of human vinculin. Structure 12: 1189-1197

Burridge K, Mangeat P (1984) An interaction between vinculin and talin. Nature 308: 744-746

Calderwood DA, Yan B, de Pereda JM, Alvarez BG, Fujioka Y, Liddington RC, Ginsberg MH (2002) The phosphotyrosine binding-like domain of talin activates integrins. J Biol Chem 277: 21749-21758

Chandrasekar I, Stradal TE, Holt MR, Entschladen F, Jockusch BM, Ziegler WH (2005) Vinculin acts as a sensor in lipid regulation of adhesion-site turnover. J Cell Sci 118: 1461-1472

Cohen DM, Chen H, Johnson RP, Choudhury B, Craig SW (2005) Two distinct head-tail interfaces cooperate to suppress activation of vinculin by talin. J Biol Chem 280: 17109-17117

Cohen DM, Kutscher B, Chen H, Murphy DB, Craig SW (2006) A conformational switch in vinculin drives formation and dynamics of a talin-vinculin complex at focal adhesions. J Biol Chem 281: 1600616015

Coll JL, Ben-Ze'ev A, Ezzell RM, Rodriguez Fernandez JL, Baribault H, Oshima RG, Adamson ED (1995) Targeted disruption of vinculin genes in F9 and embryonic stem cells changes cell morphology, adhesion, and locomotion. Proc Natl Acad Sci U S A 92: 9161-9165

Craig AM, Banker G (1994) Neuronal polarity. Annu Rev Neurosci 17: 267-310 
Demali KA (2004) Vinculin--a dynamic regulator of cell adhesion. Trends in biochemical sciences 29: 565-567

Dent EW, Gupton SL, Gertler FB (2011) The growth cone cytoskeleton in axon outgrowth and guidance. Cold Spring Harbor perspectives in biology 3

Dent EW, Kwiatkowski AV, Mebane LM, Philippar U, Barzik M, Rubinson DA, Gupton S, Van Veen JE, Furman C, Zhang J et al (2007) Filopodia are required for cortical neurite initiation. Nature cell biology 9: $1347-1359$

Ferrere A, Vitalis T, Gingras H, Gaspar P, Cases O (2006) Expression of Cux-1 and Cux-2 in the developing somatosensory cortex of normal and barrel-defective mice. Anat Rec A Discov Mol Cell Evol Biol 288: 158-165

Garcia-Alvarez B, de Pereda JM, Calderwood DA, Ulmer TS, Critchley D, Campbell ID, Ginsberg MH, Liddington RC (2003) Structural determinants of integrin recognition by talin. Molecular cell 11: 49-58

Hetrick B, Han MS, Helgeson LA, Nolen BJ (2013) Small molecules CK-666 and CK-869 inhibit actinrelated protein $2 / 3$ complex by blocking an activating conformational change. Chemistry \& biology 20: 701-712

Humphries JD, Wang P, Streuli C, Geiger B, Humphries MJ, Ballestrem C (2007) Vinculin controls focal adhesion formation by direct interactions with talin and actin. J Cell Biol 179: 1043-1057

Huttelmaier S, Bubeck P, Rudiger M, Jockusch BM (1997) Characterization of two F-actin-binding and oligomerization sites in the cell-contact protein vinculin. Eur J Biochem 247: 1136-1142

Ishikawa R, Kohama K (2007) Actin-binding proteins in nerve cell growth cones. J Pharmacol Sci 105: 6-11

Johnson RP, Craig SW (1994) An intramolecular association between the head and tail domains of vinculin modulates talin binding. J Biol Chem 269: 12611-12619

Johnson RP, Craig SW (1995) F-actin binding site masked by the intramolecular association of vinculin head and tail domains. Nature 373: 261-264

Kwiatkowski AV, Rubinson DA, Dent EW, Edward van Veen J, Leslie JD, Zhang J, Mebane LM, Philippar U, Pinheiro EM, Burds AA et al (2007) Ena/VASP Is Required for neuritogenesis in the developing cortex. Neuron 56: 441-455

Le Clainche C, Dwivedi SP, Didry D, Carlier MF (2010) Vinculin is a dually regulated actin filament barbed end-capping and side-binding protein. J Biol Chem 285: 23420-23432 
Le S, Hu X, Yao M, Chen H, Yu M, Xu X, Nakazawa N, Margadant FM, Sheetz MP, Yan J (2017) Mechanotransmission and Mechanosensing of Human alpha-Actinin 1. Cell reports 21: 2714-2723

Letourneau PC, Pech IV, Rogers SL, Palm SL, McCarthy JB, Furcht LT (1988) Growth cone migration across extracellular matrix components depends on integrin, but migration across glioma cells does not. Journal of neuroscience research 21: 286-297

Letourneau PC, Shattuck TA (1989) Distribution and possible interactions of actin-associated proteins and cell adhesion molecules of nerve growth cones. Development 105: 505-519

Li CL, Sathyamurthy A, Oldenborg A, Tank D, Ramanan N (2014) SRF phosphorylation by glycogen synthase kinase-3 promotes axon growth in hippocampal neurons. J Neurosci 34: 4027-4042

Lian G, Sheen VL (2015) Cytoskeletal proteins in cortical development and disease: actin associated proteins in periventricular heterotopia. Front Cell Neurosci 9: 99

Luo L (2002) Actin cytoskeleton regulation in neuronal morphogenesis and structural plasticity. Annual review of cell and developmental biology 18: 601-635

Menkel AR, Kroemker M, Bubeck P, Ronsiek M, Nikolai G, Jockusch BM (1994) Characterization of an F-actin-binding domain in the cytoskeletal protein vinculin. J Cell Biol 126: 1231-1240

Mierke CT (2009) The role of vinculin in the regulation of the mechanical properties of cells. Cell Biochem Biophys 53: 115-126

Mierke CT, Kollmannsberger P, Zitterbart DP, Smith J, Fabry B, Goldmann WH (2008) Mechanocoupling and regulation of contractility by the vinculin tail domain. Biophys $J$ 94: 661-670

Mullins RD, Heuser JA, Pollard TD (1998) The interaction of Arp2/3 complex with actin: nucleation, high affinity pointed end capping, and formation of branching networks of filaments. Proceedings of the National Academy of Sciences of the United States of America 95: 6181-6186

Nadarajah B, Parnavelas JG (2002) Modes of neuronal migration in the developing cerebral cortex. Nat Rev Neurosci 3: 423-432

Nair D, Hosy E, Petersen JD, Constals A, Giannone G, Choquet D, Sibarita JB (2013) Super-resolution imaging reveals that AMPA receptors inside synapses are dynamically organized in nanodomains regulated by PSD95. The Journal of neuroscience : the official journal of the Society for Neuroscience 33: 13204-13224

Riedl J, Crevenna AH, Kessenbrock K, Yu JH, Neukirchen D, Bista M, Bradke F, Jenne D, Holak TA, Werb Z et al (2008) Lifeact: a versatile marker to visualize F-actin. Nature methods 5: 605-607 
Roca-Cusachs P, del Rio A, Puklin-Faucher E, Gauthier NC, Biais N, Sheetz MP (2013) Integrindependent force transmission to the extracellular matrix by alpha-actinin triggers adhesion maturation. Proceedings of the National Academy of Sciences of the United States of America 110: E1361-1370

Romero S, Le Clainche C, Gautreau AM (2020) Actin polymerization downstream of integrins: signaling pathways and mechanotransduction. Biochem J 477: 1-21

Sturner T, Tatarnikova A, Mueller J, Schaffran B, Cuntz H, Zhang Y, Nemethova M, Bogdan S, Small V, Tavosanis G (2019) Transient localization of the Arp2/3 complex initiates neuronal dendrite branching in vivo. Development 146

Sydor AM, Su AL, Wang FS, Xu A, Jay DG (1996) Talin and vinculin play distinct roles in filopodial motility in the neuronal growth cone. J Cell Biol 134: 1197-1207

Thievessen I, Thompson PM, Berlemont S, Plevock KM, Plotnikov SV, Zemljic-Harpf A, Ross RS, Davidson MW, Danuser G, Campbell SL et al (2013) Vinculin-actin interaction couples actin retrograde flow to focal adhesions, but is dispensable for focal adhesion growth. J Cell Biol 202: 163-177

Thompson PM, Tolbert CE, Shen K, Kota P, Palmer SM, Plevock KM, Orlova A, Galkin VE, Burridge $\mathrm{K}$, Egelman EH et al (2014) Identification of an actin binding surface on vinculin that mediates mechanical cell and focal adhesion properties. Structure 22: 697-706

Varnum-Finney B, Reichardt LF (1994) Vinculin-deficient PC12 cell lines extend unstable lamellipodia and filopodia and have a reduced rate of neurite outgrowth. J Cell Biol 127: 1071-1084

Xu W, Baribault H, Adamson ED (1998a) Vinculin knockout results in heart and brain defects during embryonic development. Development 125: 327-337

$\mathrm{Xu}$ W, Coll JL, Adamson ED (1998b) Rescue of the mutant phenotype by reexpression of full-length vinculin in null F9 cells; effects on cell locomotion by domain deleted vinculin. J Cell Sci 111 ( Pt 11): $1535-1544$

Zhang M, Chang H, Zhang Y, Yu J, Wu L, Ji W, Chen J, Liu B, Lu J, Liu Y et al (2012) Rational design of true monomeric and bright photoactivatable fluorescent proteins. Nature methods 9: 727-729

Ziegler WH, Liddington RC, Critchley DR (2006) The structure and regulation of vinculin. Trends Cell Biol 16: 453-460 


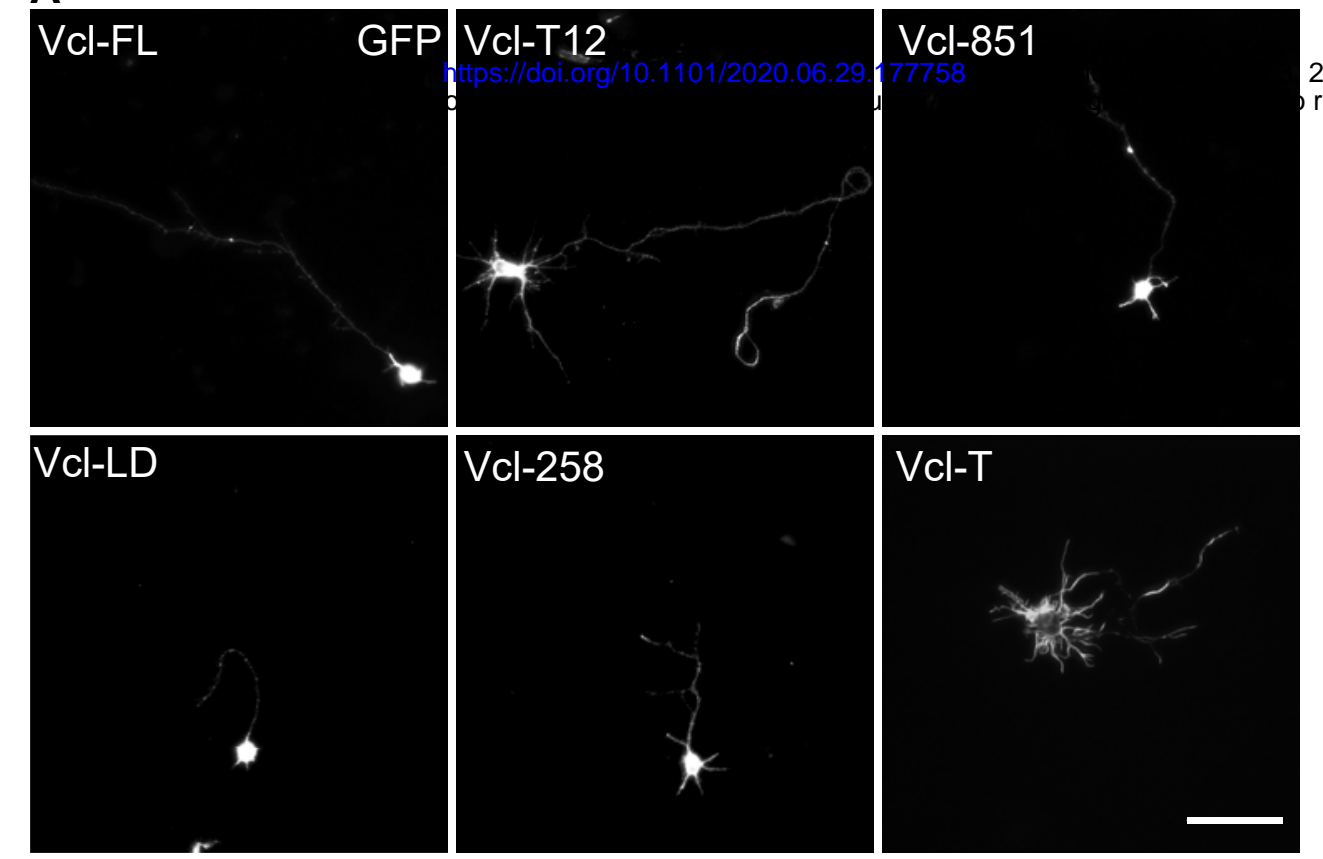

2020. The copyright holder for this preprint (which reuse allo 8 edowithout permission.

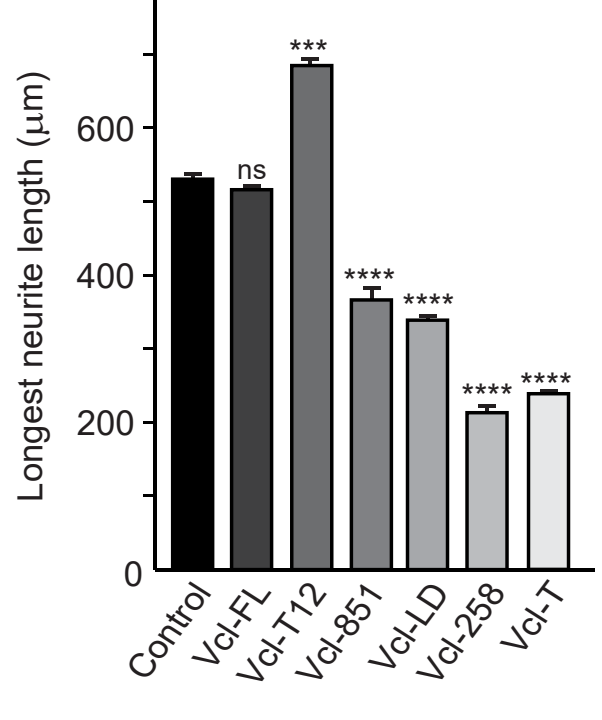

C

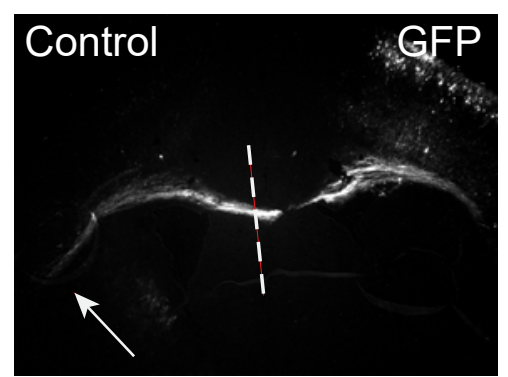

Vcl-T12
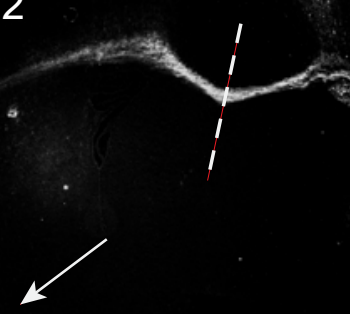

Vcl-T

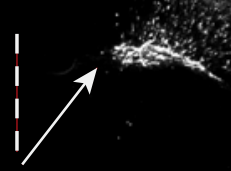

\section{GFP}

E
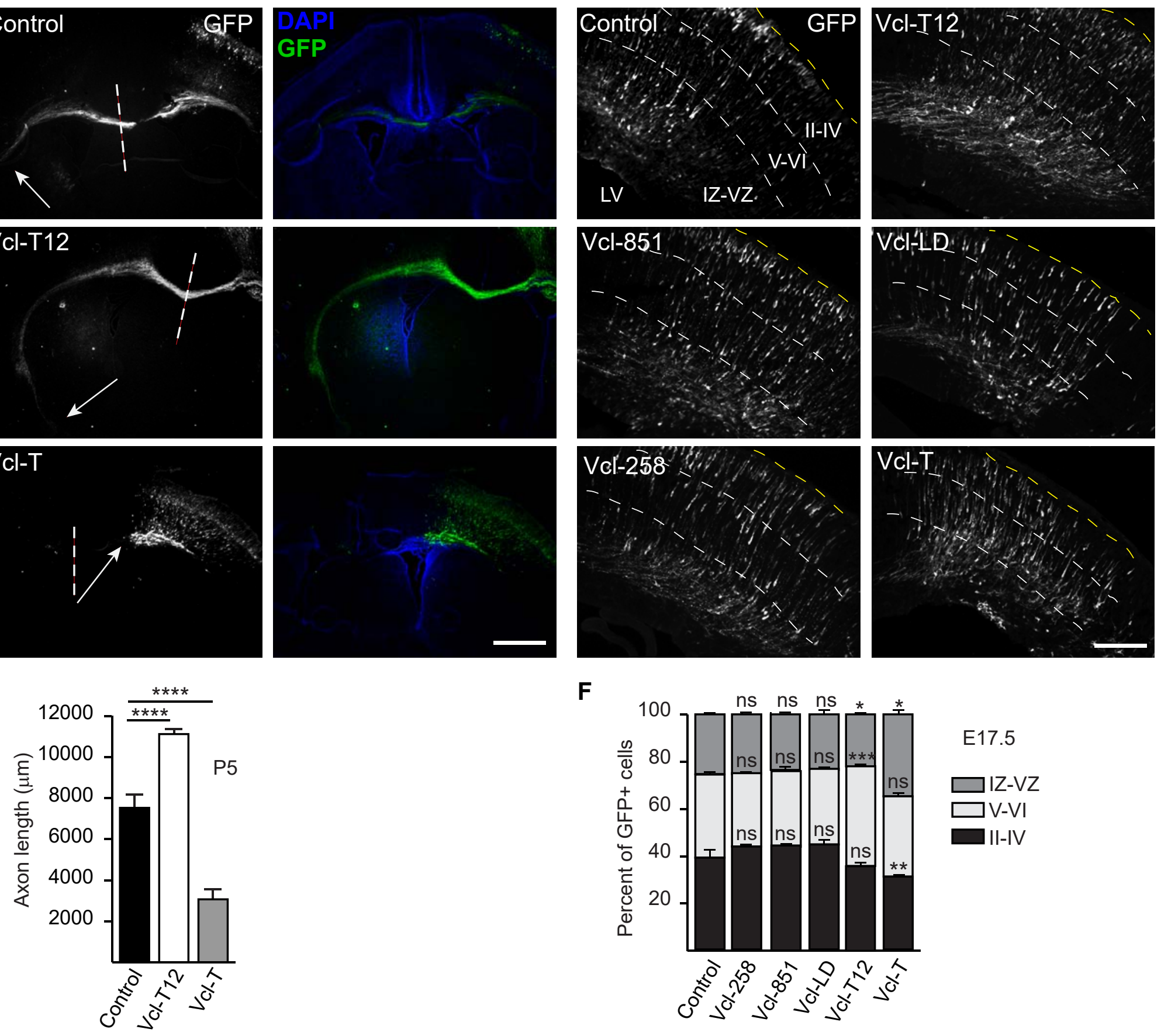

F

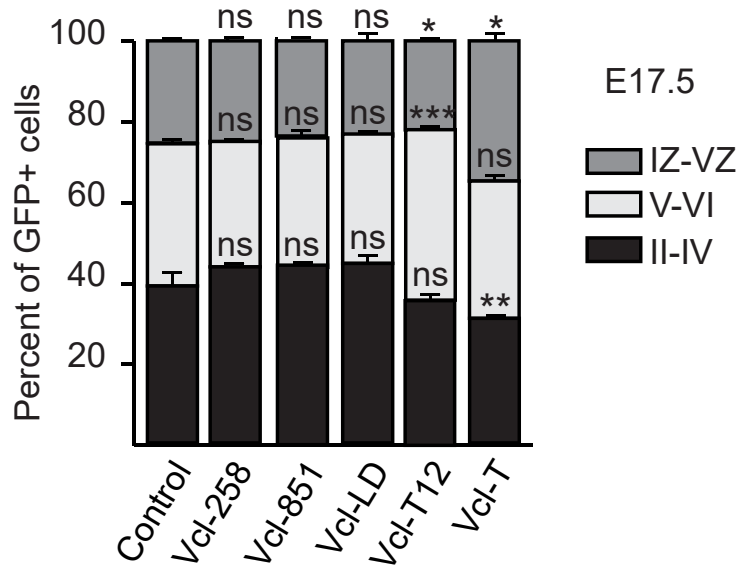


A

bioRxiv preprint doi: https://doi.org/10.1101/2020.06.29.177758; this version posted July 3, 2020. The copyright holder for this preprint (Fiłeldre 2

\section{GFP}
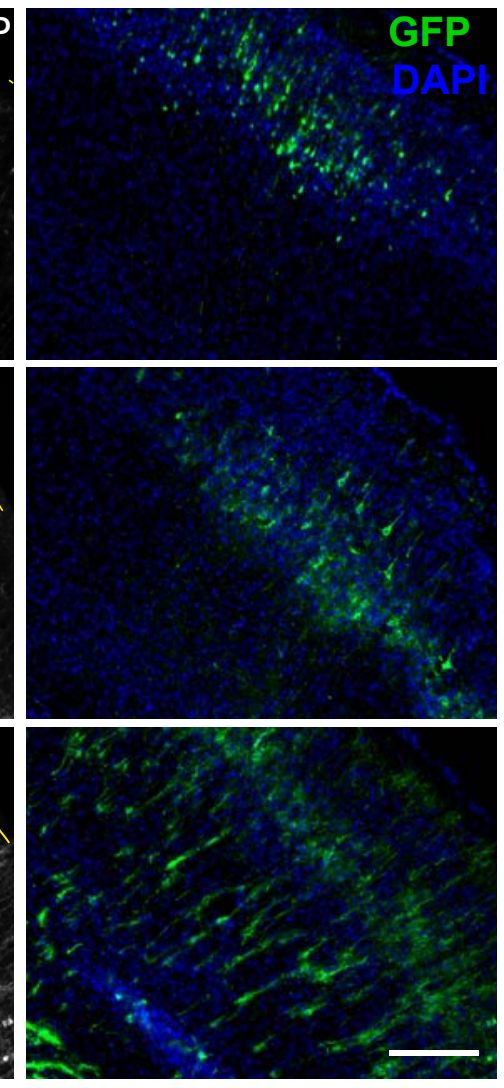

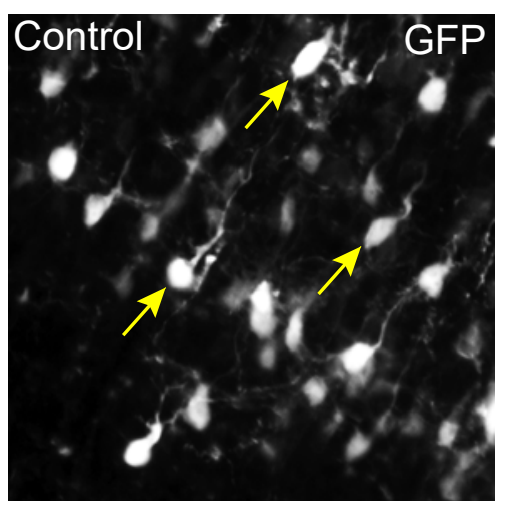

B

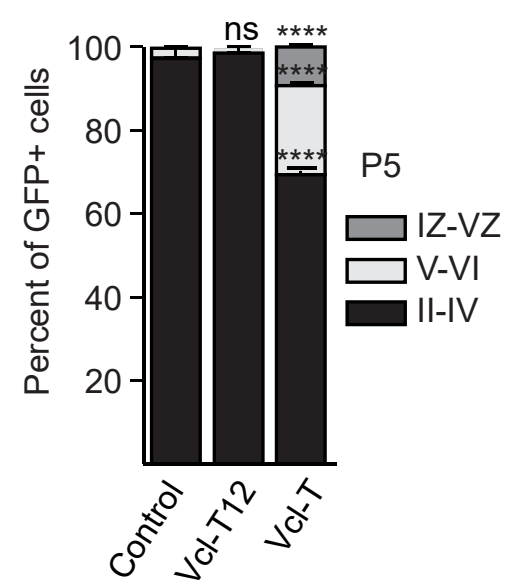

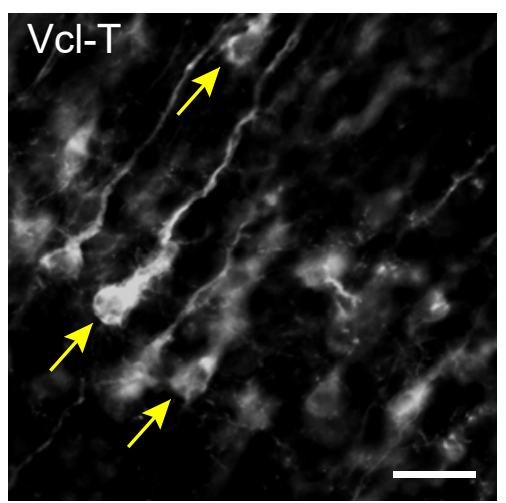

D $\quad 1500$ P5

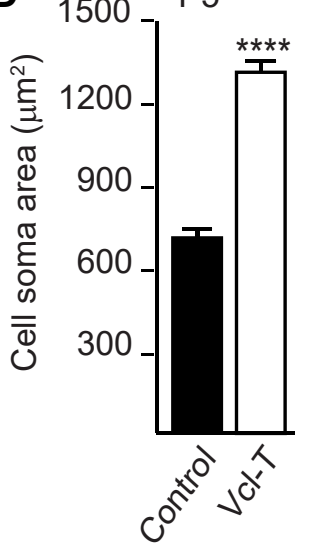

E
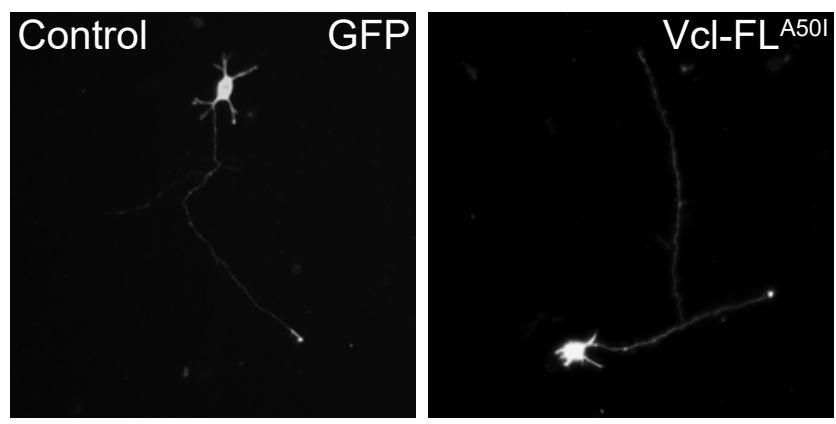$$
\text { Vcl-851 }{ }^{\text {A50I }}
$$

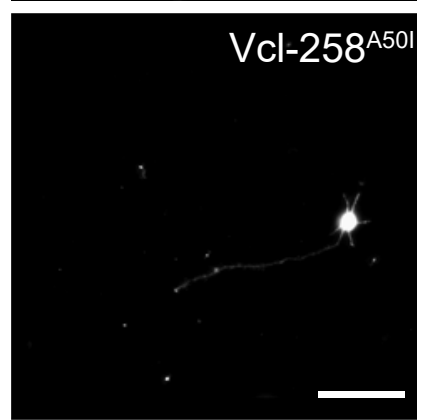

F

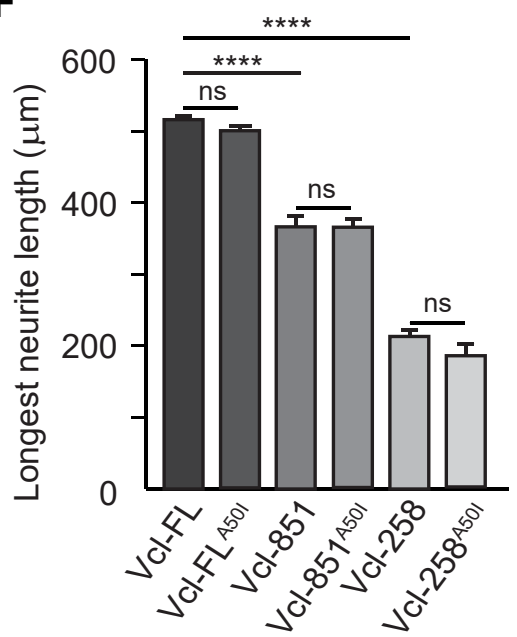

G

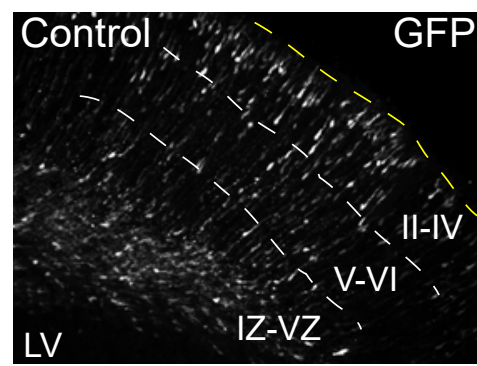

H

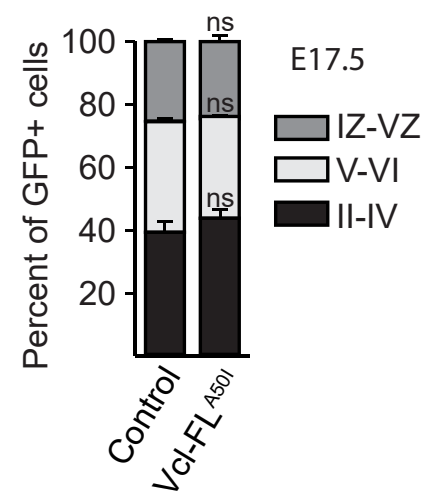


Figure 3

A
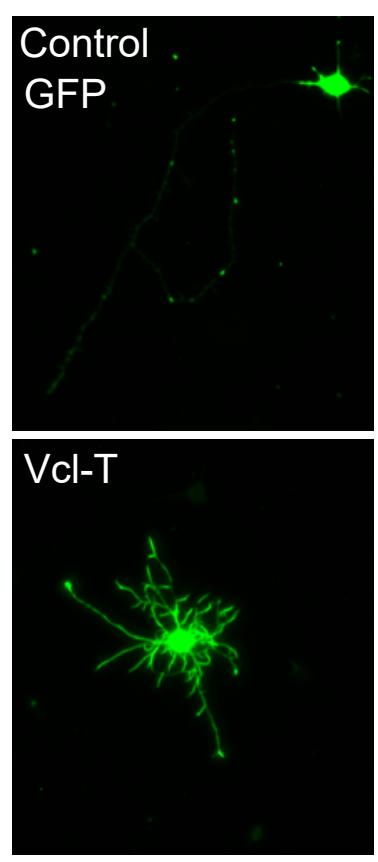

C
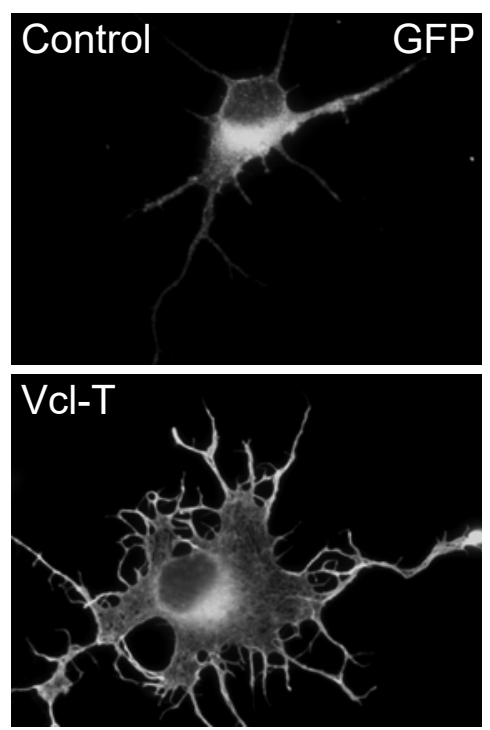

E

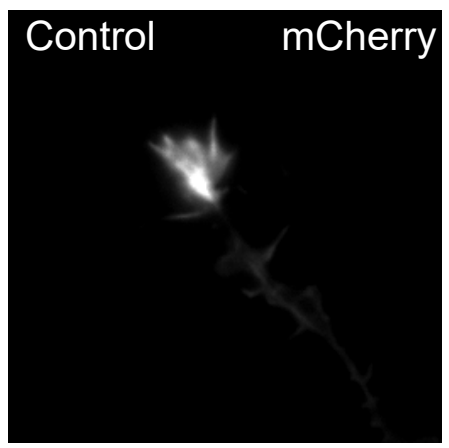

Tau
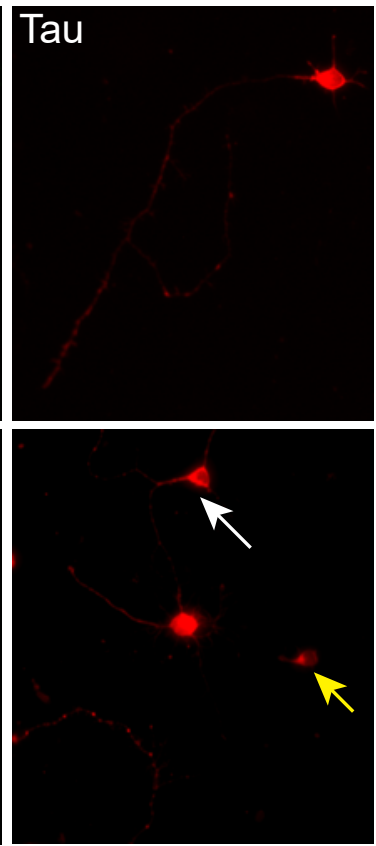

R
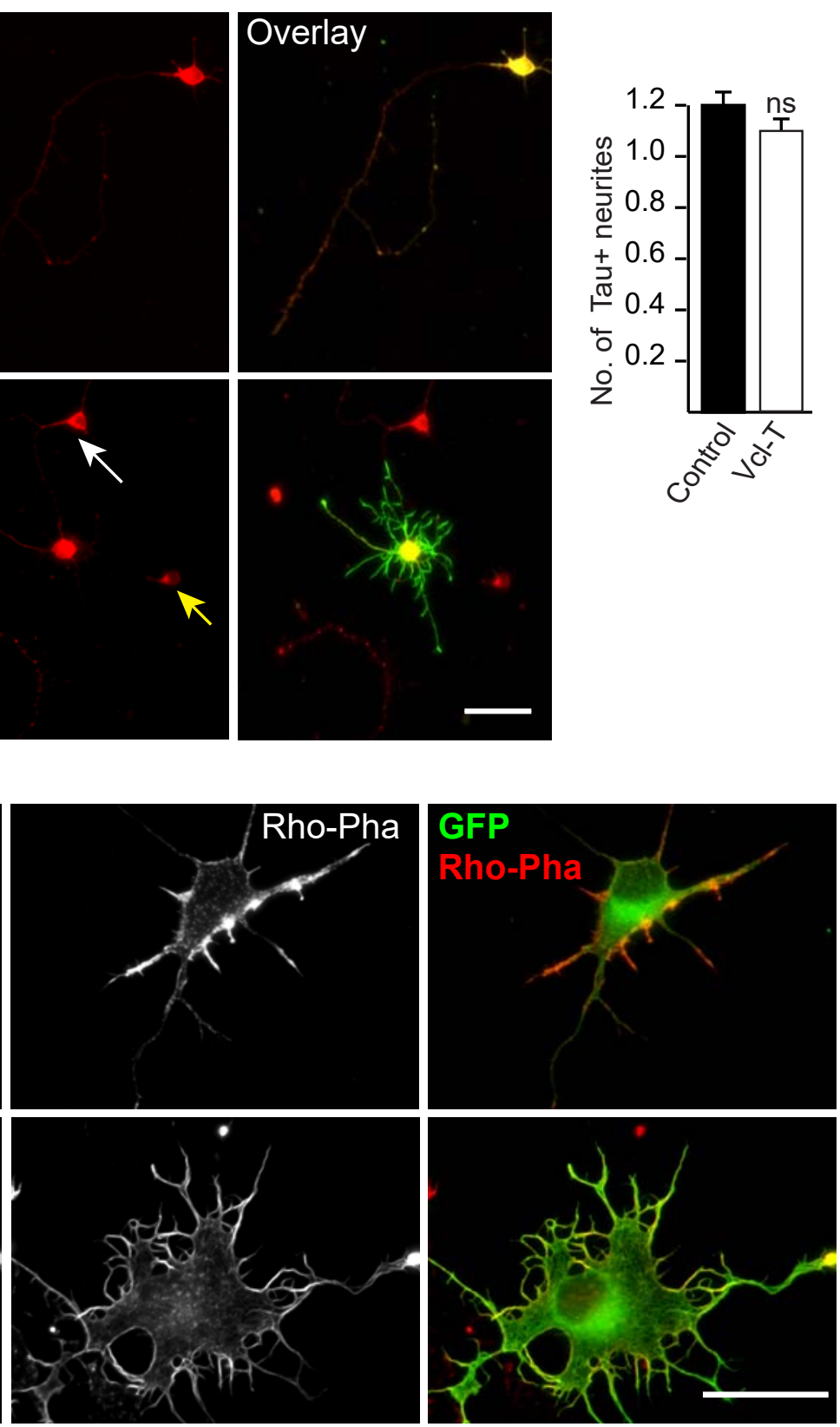

F
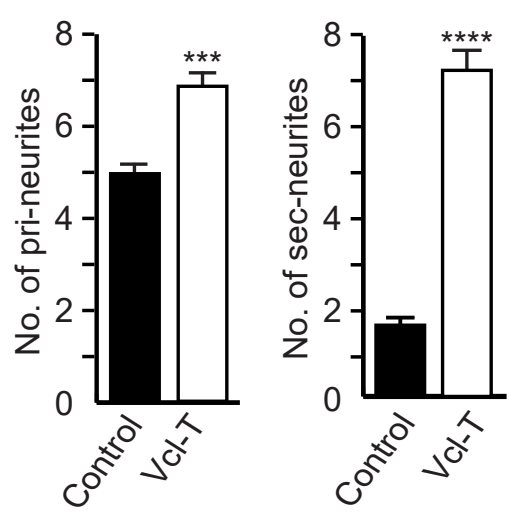

D

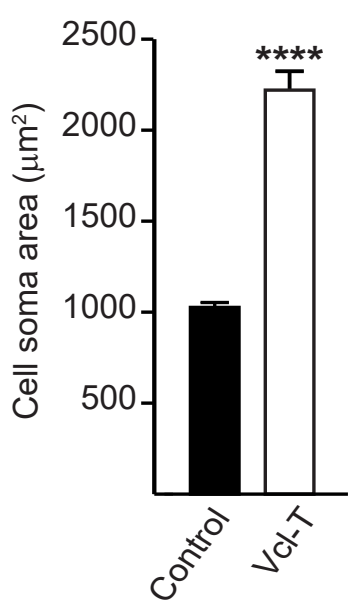

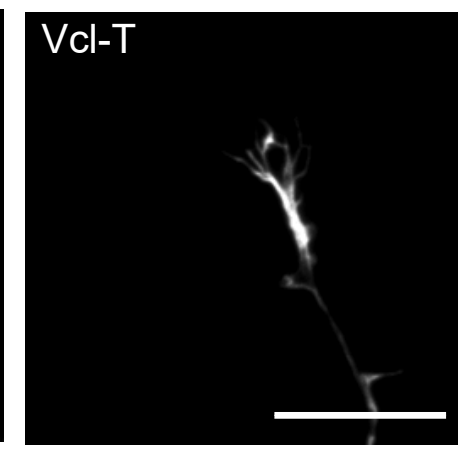

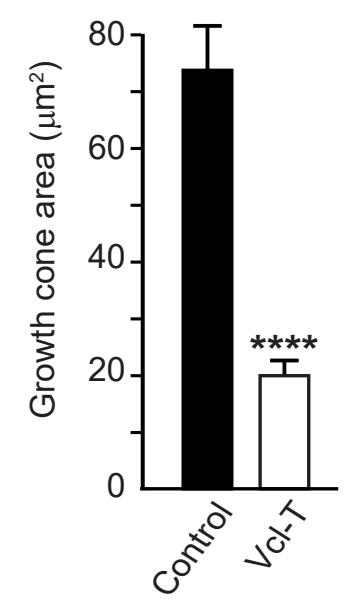


A

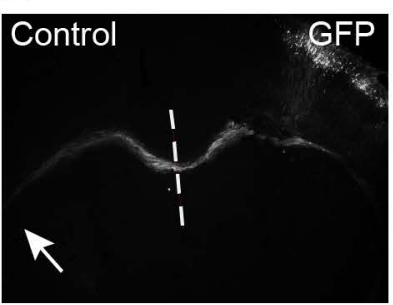

\section{Vcl-T}

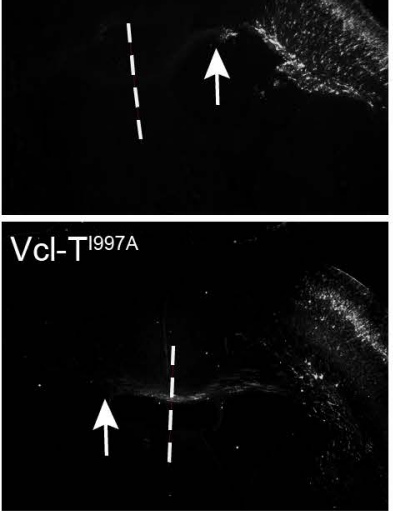

E

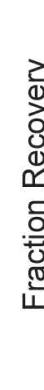

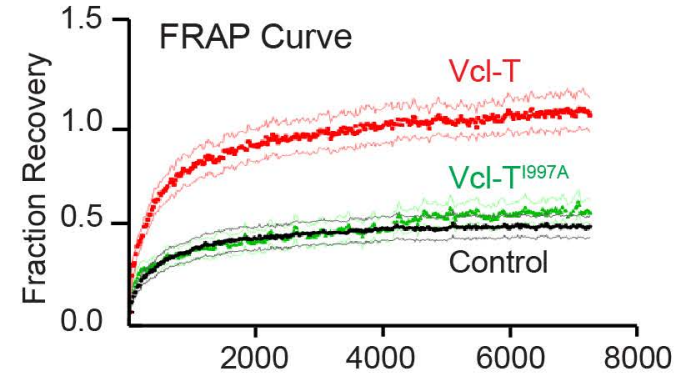

H

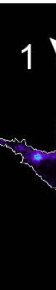

$\downarrow$

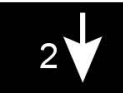

1
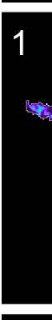
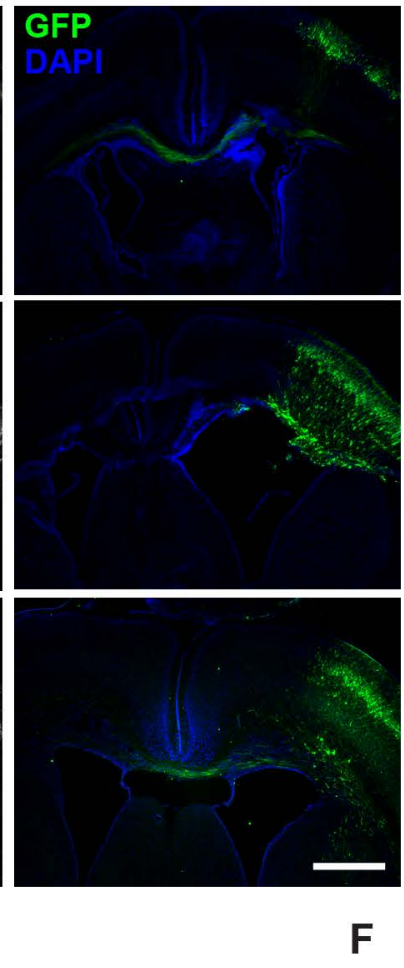

F

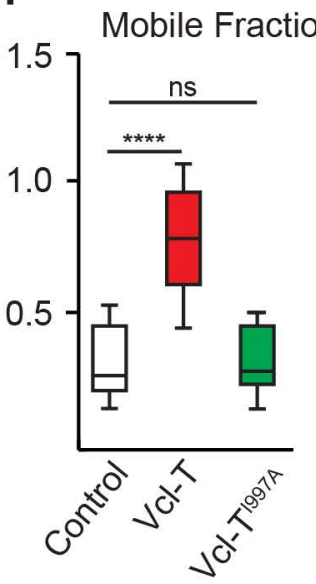

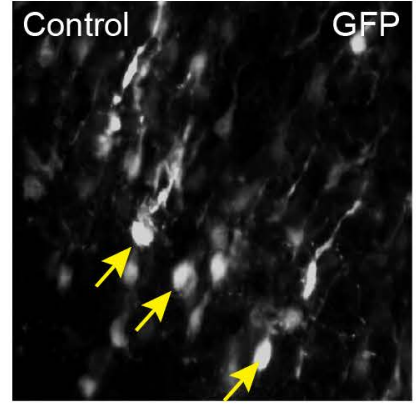

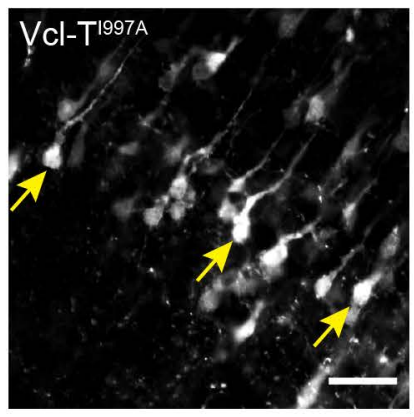

G

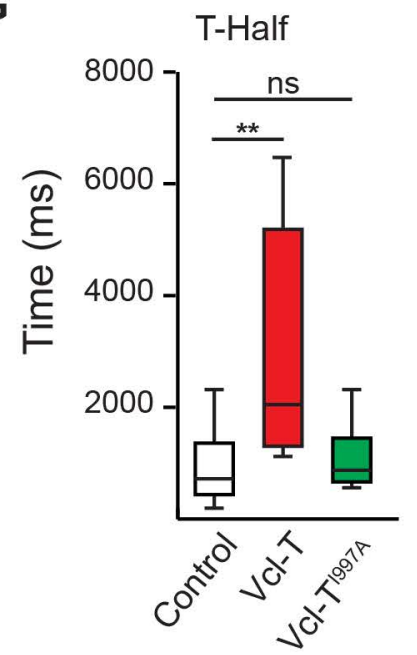

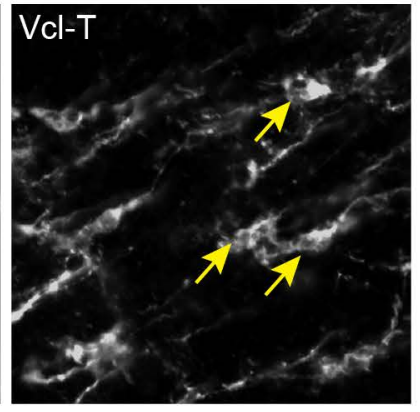

D
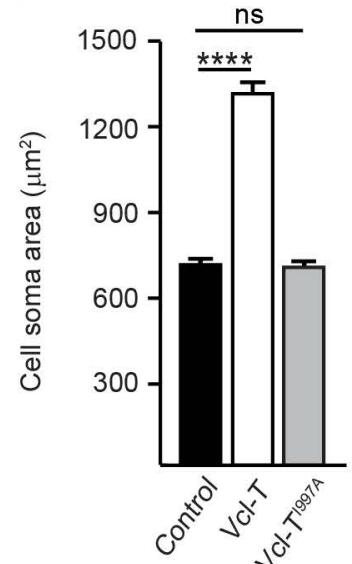

J

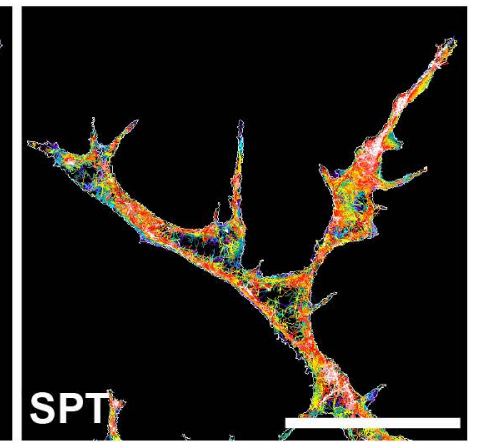

I
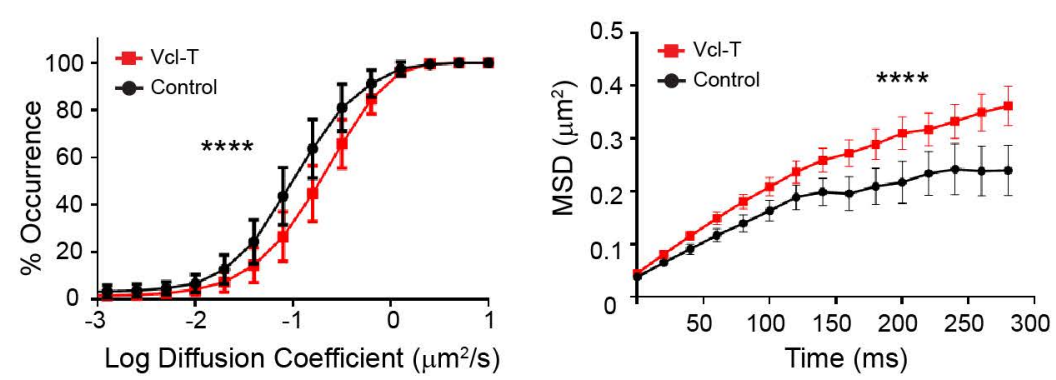

K

L

M
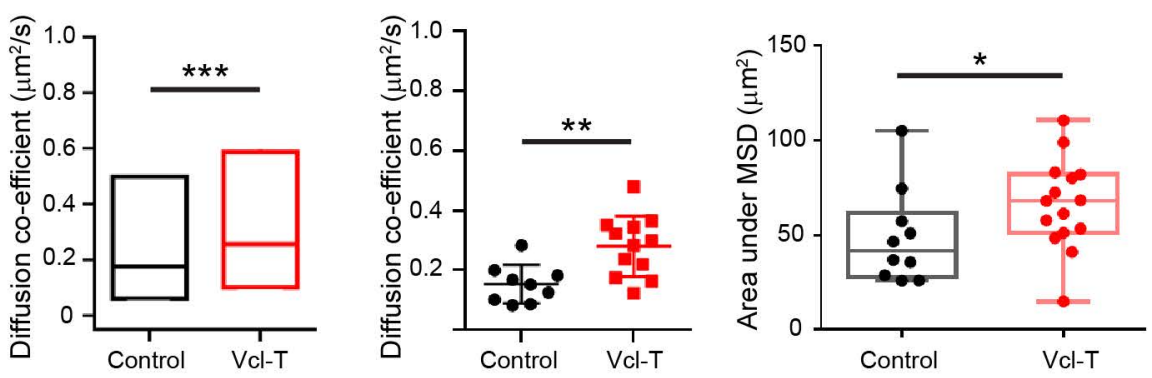
bioRxiv preprint doi: https://doi.org/10.1101/2020.06.29.177758; this version posted July 3, 2020. The copyright holder for this preprint (which was not certified by peer review) is the author/funder. All rights reserved. No reuse allowed without permission.

\section{Supplementary Figure Legends}

Suppl. Figure 1. Vinculin deletion attenuated axon growth and enhanced neuronal migration. (A) NIH3T3 cells were transfected with a pair of single guide RNAs (gRNA) targeting Vinculin along with Cas9n nickase and cells were lysed at $72 \mathrm{hrs}$ post-transfection. Western blot showing reduction in vinculin expression in Vcl-gRNA transfected cells (Vcl$\mathrm{KO}$ ). Actin was used for loading control. (B) Quantification of western blot signals from (A). (C) Primary neocortical neurons were electroporated with either empty Cas9n-T2A-GFP plasmid or Vcl-gRNA-Cas9n-T2A-GFP construct. Cells were fixed at 4 DIV and immunostained for GFP for transfected neurons and $\beta$-III-tubulin (Tuj-1) to visualize neurites. Neurons expressing Vcl-gRNAs exhibited shorter neurites compared to control neurons. Nontransfected (arrows) and Vcl-gRNA expressing cells (arrowhead) are indicated. (D) Quantification of longest neurite length from (C) ( $\mathrm{n}=100$ cells). (E) Neuronal progenitors at E14.5 were electroporated with either empty GFP (control) vector or Vcl-gRNA construct at E14.5 and analysed at E17.5 showed that Vcl-KO stunts axonal growth in vivo as well. Arrows indicate the axon terminals along corpus callosum. Inset shows magnified view of the axonal fibre. (F) Quantification of axon length from (E) ( $\mathrm{n}=3$ animals). (G) Embryos were electroporated at E14.5 with Vcl-gRNAs and analysed at E17.5. Vinculin deletion enhances neuronal migration during corticogenesis. (H) Quantification of the percentage of $\mathrm{GFP}^{+}$ neurons in different layers of cortex shown in $(\mathrm{G})(\mathrm{n}=3$ animals). (I) Analysis at E17.5 also showed that vinculin deletion stunts leading processes of neurons. (J) Quantification of the length of leading processes from (I) ( $\mathrm{n}=200$ cells, $\mathrm{n}=3$ animals). Scale $50 \mu \mathrm{m}(\mathrm{C}), 2 \mathrm{~mm}(\mathrm{E})$, $150 \mu \mathrm{m}$ (inset in E), $400 \mu \mathrm{m}(\mathrm{G}), 100 \mu \mathrm{m}$ in (I), *** $p<0.001$, **** $p<0.0001$. ns, non-significant. Two-tailed student's $t$ test (D, F, J). One-way ANOVA and Tukey's post-hoc test (H).

Suppl. Figure 2. (A) Cartoon showing the partial vector map of full-length vinculin tagged to GFP sequence at the 5' terminus and driven by CAG promoter. (B) Cartoon depicting different mutant clones of vinculin constructed and tested. Red vertical lines indicate the positions of point mutations.

Suppl. Figure 3. Vcl-T expression affects neocortical lamination. (A) Embryos were electroporated at E14.5 with control GFP plasmid or Vcl-T and analysed at P5. 20- $\mu \mathrm{m}$ brains sections were immunostained for GFP and layer 2-4 specific marker, Cux1. In control sections, all $\mathrm{Cux} 1^{+}$neurons had reached the upper layers as expected. In contrast, the Vcl-T transfected embryos, many $\mathrm{Cux}^{+}$neurons were seen occupying the lower layers including around the ventricular zone/intermediate zone (VZ/IZ). Scale, $400 \mu \mathrm{m}$. (B) Quantification of the number of $\mathrm{Cux}^{+}$cells in bottom layers of cortex (layers V \& VI) and in IZ/VZ from (E) (n=3 animals, $* * * p<0.001$. Two-tailed student's $t$ test.

Suppl. Figure 4. Vcl-T increases branching early in development in vitro. Cortical neurons from neonatal mice were transfected with control plasmid and Vcl-T and plated. Cells were fixed at $24 \mathrm{~h} \mathrm{(A)}$ and $48 \mathrm{~h}$ (C) and visualized for GFP expression. Vcl-T expressing neurons had a greater number of both primary and secondary neurites even at $24 \mathrm{hrs}$ post-plating. Scale, $100 \mu \mathrm{m})$. (B, D) Quantification of the length of longest neurite, number of primary and secondary neurites at $24 \mathrm{hrs}$ (B) and $48 \mathrm{hrs}$ (D) ( $\mathrm{n}=100$ cells, $* * * * p<0.0001$. ns, nonsignificant). Two-tailed student's $t$ test. 
bioRxiv preprint doi: https://doi.org/10.1101/2020.06.29.177758; this version posted July 3, 2020. The copyright holder for this preprint (which was not certified by peer review) is the author/funder. All rights reserved. No reuse allowed without permission.

Suppl. Figure 5. Abolishing actin-Vcl-T interaction reverses cell soma but not growth cone morphology. (A) Representative images of neocortical neurons transfected with control vector, Vcl-T and $\mathrm{Vcl}-\mathrm{T}^{\mathrm{T} 997 \mathrm{~A}}$ constructs and stained for F-actin using rhodamine-conjugated phalloidin (Rho-Pha). Abolishing interaction between $\mathrm{Vcl}-\mathrm{T}$ and actin $\left(\mathrm{Vcl}_{-} \mathrm{T}^{\mathrm{I} 997 \mathrm{~A}}\right)$ reduced the cell soma area to that seen in control neurons. (B) Quantification of the cell soma area in control, Vcl-T and Vcl-T ${ }^{1997 A}$ expressing neurons shown in (A) ( $\mathrm{n}=100$ cells). (C) Representative images from live imaging of control $\mathrm{Vcl}-\mathrm{T}$ and $\mathrm{Vcl}^{-\mathrm{T}^{1977 \mathrm{~A}}}$ expressing neurons co-transfected with LifeAct-mCherry to visualize actin. Abolishing actin-Vcl-T interaction does not rescue growth cone defect exhibited by Vcl-T. (D) Quantification of the growth cone area in control, Vcl-T and Vcl-T ${ }^{1997 A}$ expressing neurons shown in (C) $(n=25$ cells). Scale, $50 \mu \mathrm{m}(\mathrm{A}), 10 \mu \mathrm{m}(\mathrm{C}) .{ }^{* * * *} p<0.0001$; ns, non-significant. Two-tailed student's $t$ test.

Suppl. Figure 6. (A) Vinculin full-length defective in actin binding (Vcl-FL ${ }^{1997 A}$ ) does not affect branching. Representative images of neurons electroporated with either empty GFP (control) vector or vinculin full-length defective in actin binding $\left(\mathrm{Vcl}_{-} \mathrm{FL}^{1997 \mathrm{~A}}\right)$. Scale, $100 \mu \mathrm{m}$. (B) Quantification of longest neurite length and number of primary and secondary neurites from neurons shown in (A) $(\mathrm{n}=100$ cells). $* * * * p<0.0001$; ns, non-significant. Two-tailed student's $t$ test.

Suppl. Figure 7. Abolishing actin-Vcl-T interaction rescues cortical lamination defect caused by Vcl-T. (A) Analysis of cortical lamination using Cux1 immunostaining shows the rescue of migration of upper layer neurons when actin-Vcl-T interaction is abolished. Scale, $400 \mu \mathrm{m}$. (F) Quantification of the number of $\mathrm{Cux}^{+}{ }^{+}$cells in bottom layers of cortex (layer $\mathrm{V} \&$ $\mathrm{VI})$ and in IZ/VZ from (A) ( $\mathrm{n}=3$ animals) $* * * p<0.001$; ns, non-significant. Two-tailed student's t test. 
Suppl. Figure 1

A bioRxiv preprist doi: https://doi.org/1 1 1101/2020.06.29.177758; this version posted July 3, 2020. The copyright holder fofthis preprint (which

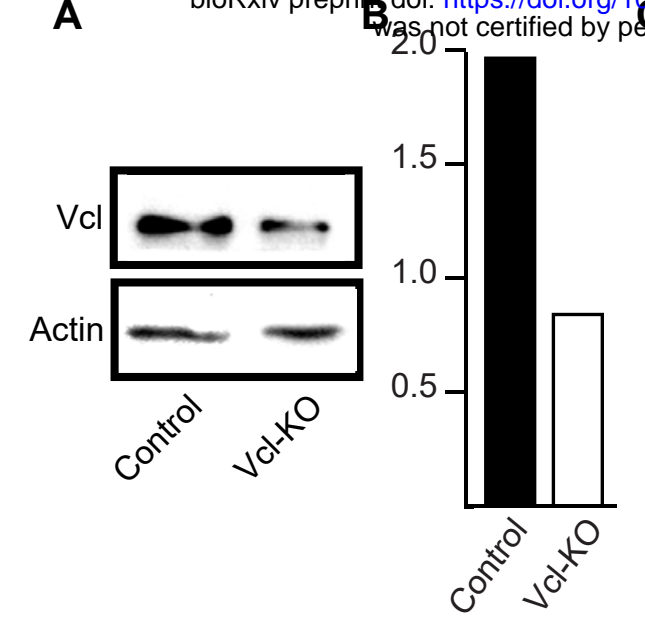

E

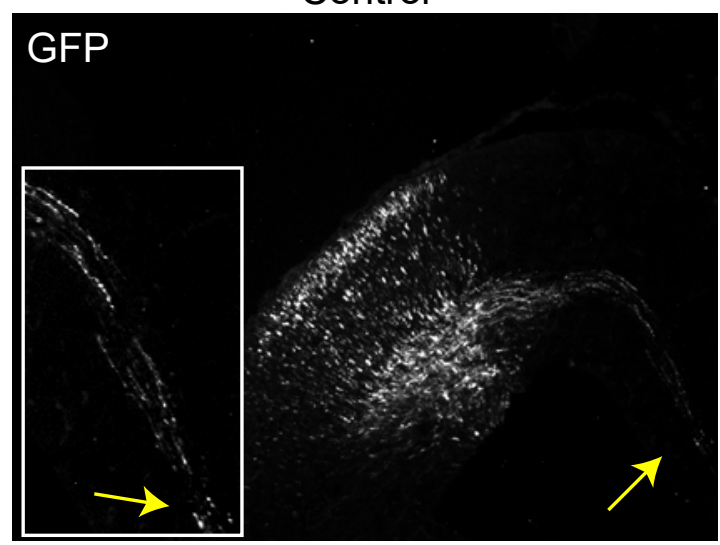

G

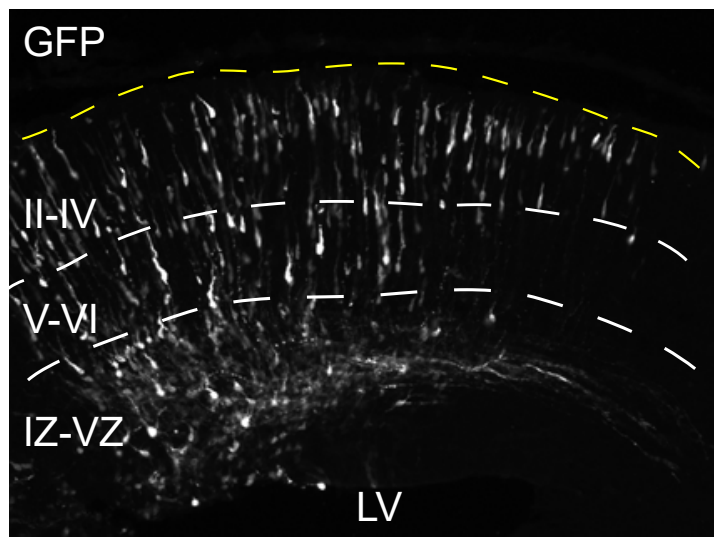

I

\section{GFP}

Control

$\mathrm{Vcl}-\mathrm{KO}$

Vcl-KO
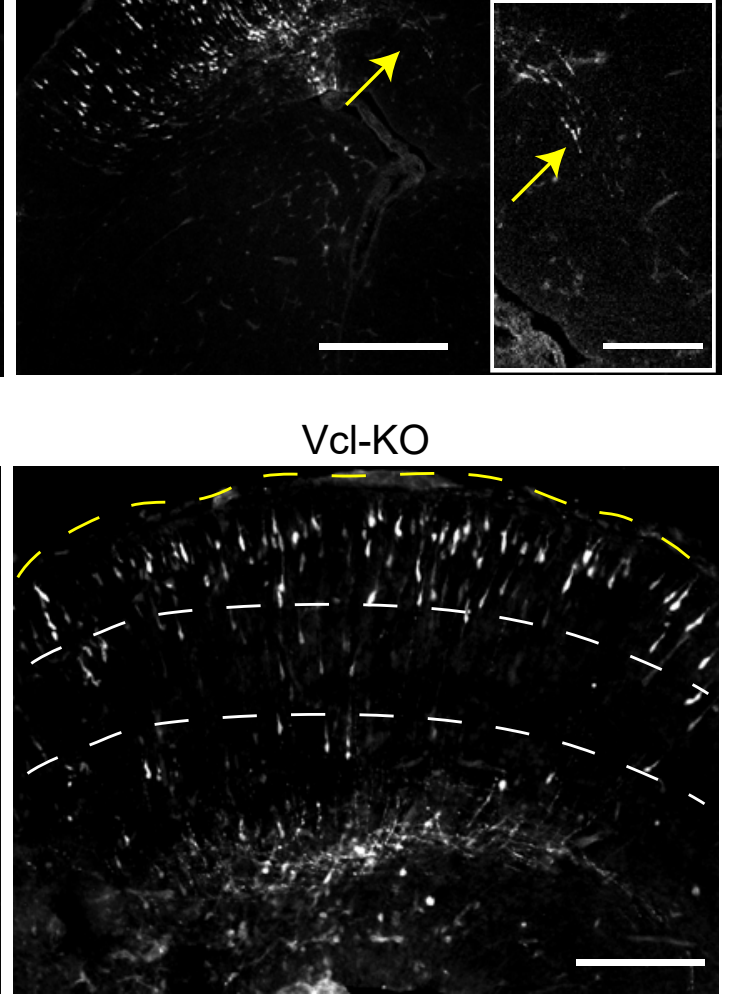

H

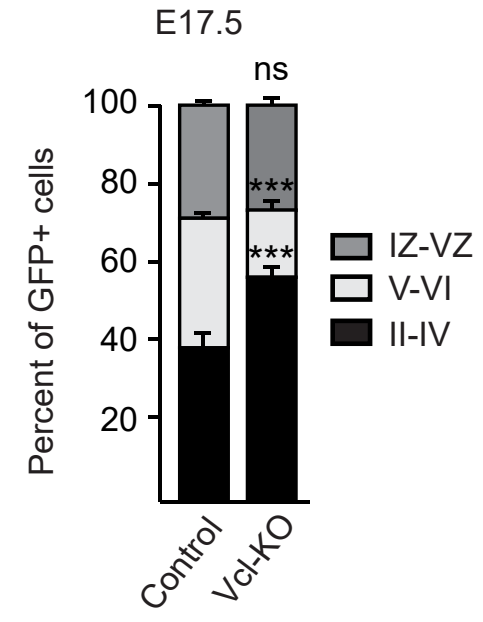


A

bioRxiv preprint doi: https://doi.org/10.1101/2020.06.29.177758; this version posted July 3, 2020. The copyright holder for this ppeprint ( was not certified by peer review) is the author/funder. All rights reserved. No reuse allowed without permission.

\begin{tabular}{l|l|l|l|} 
CAG pro & Head & Neck & Tail \\
\hline CMV enhancer GFP & \multicolumn{3}{|c|}{ Vinculin }
\end{tabular}

B

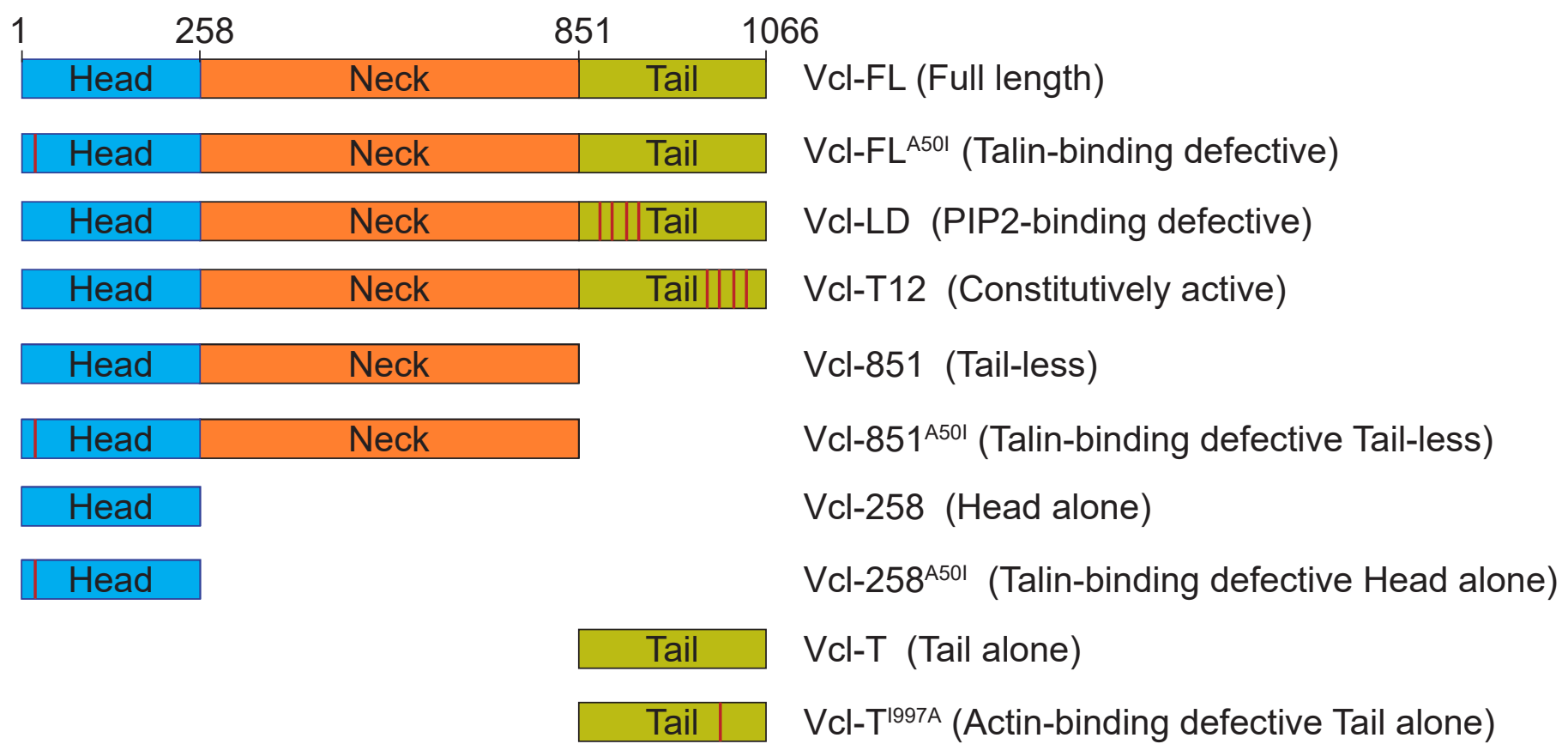


$\underset{\text { Suppl. Fiqure } 3}{\text { Spreprint (whien }}$

bioRxiv preprint doi: https://doi.org/10.1101/2020.06.29.177758; this version posted July 3, 2020. The copyright holder for this preprint. (whigureh was not certified by peer review) is the author/funder. All rights reserved. No reuse allowed without permission.

A
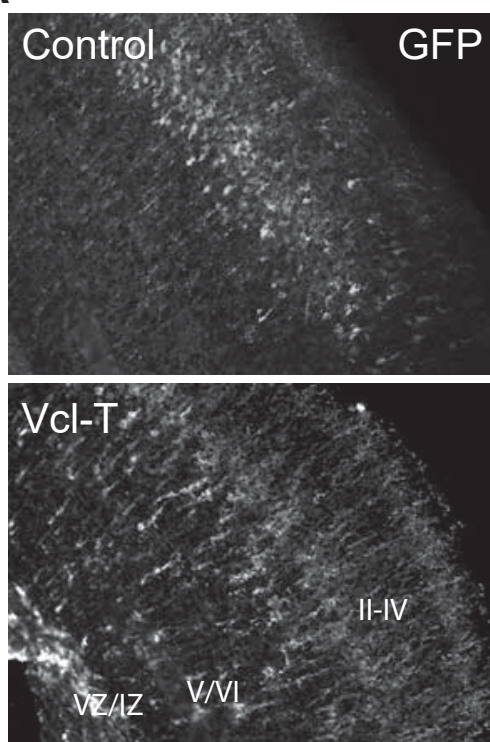

B
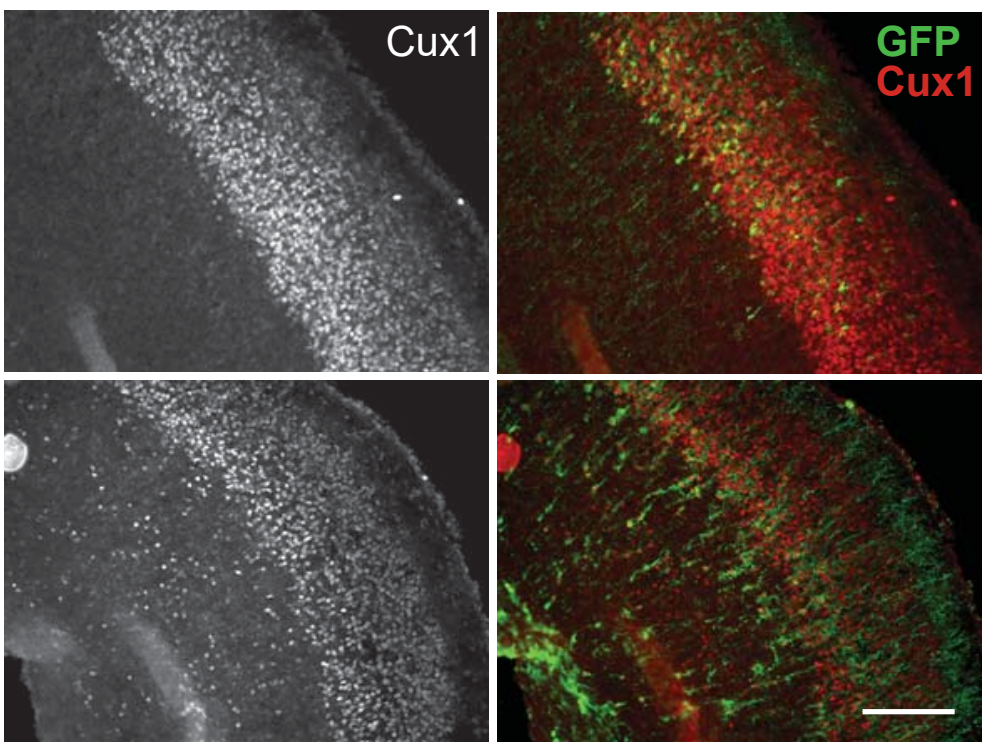

P5

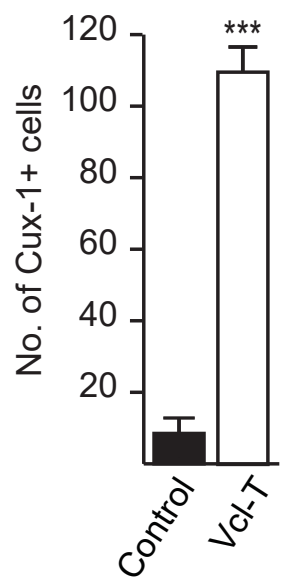


bioRxiv preprint doi: https://doi.org/10.1101/2020.06.29.177758; this version posted July 3, 2020. The copyright holder for this preprint (which was not certified by peer review) is the author/funder. All rights reserved. No reuse allowed without permission.

A

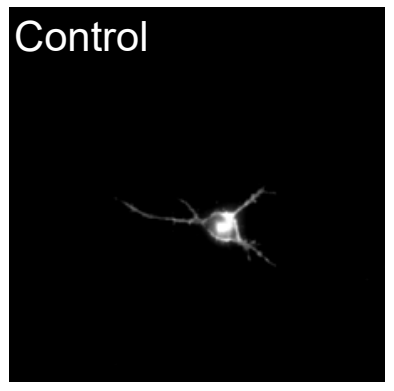

24 Hours

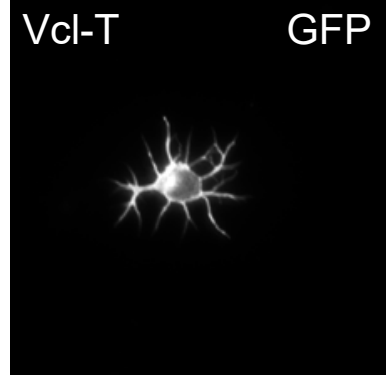

C

48 Hours

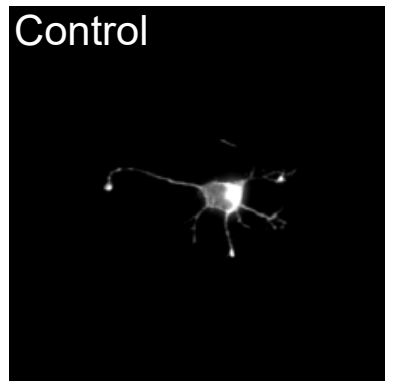

B

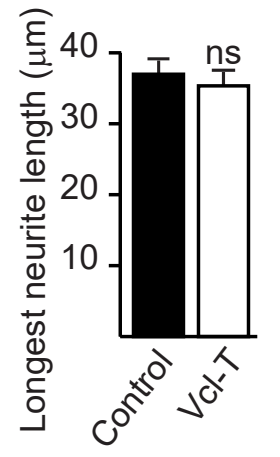

D

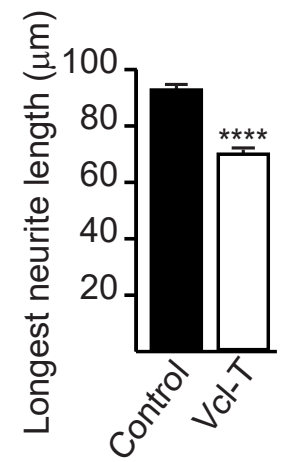

24 hrs

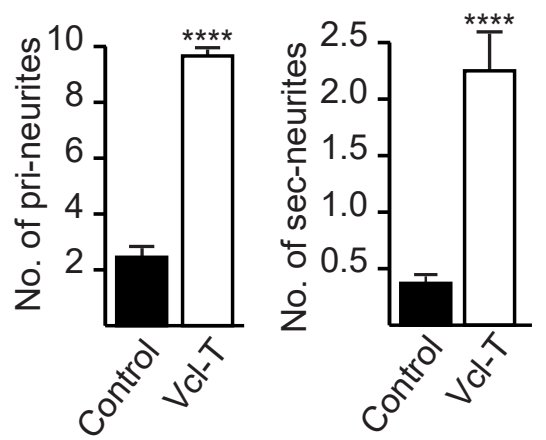

48 hrs

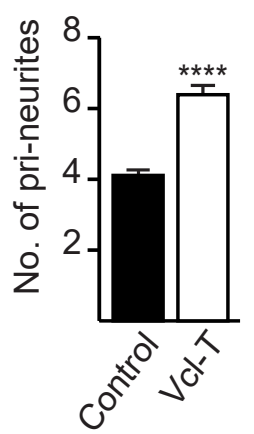

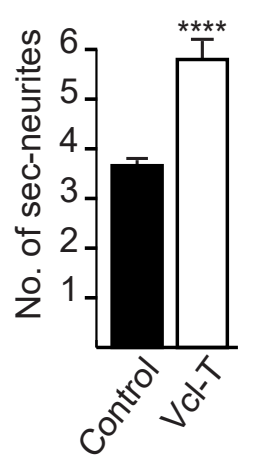


Suppl. Figure 5

A
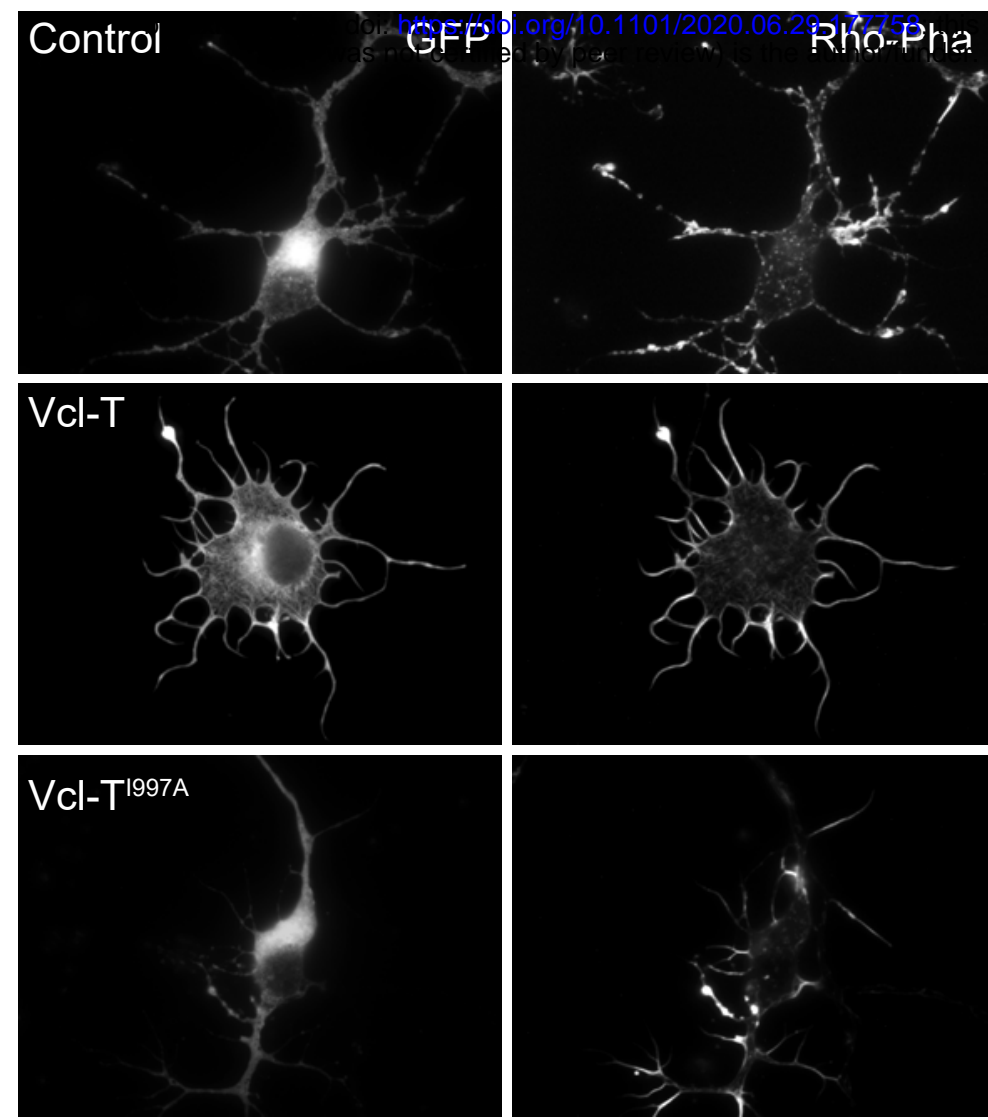

C

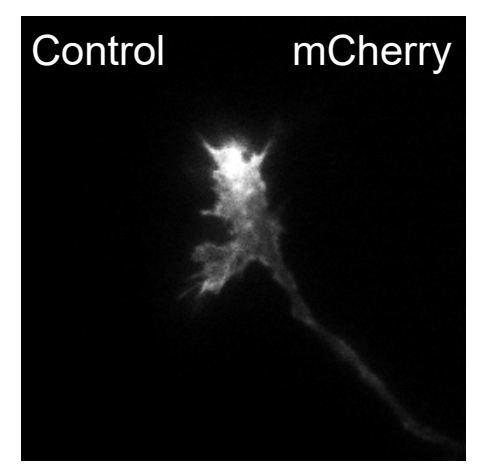

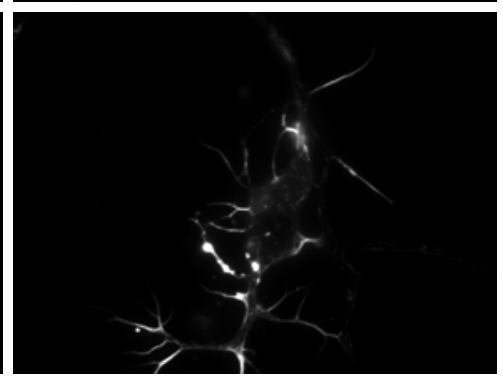
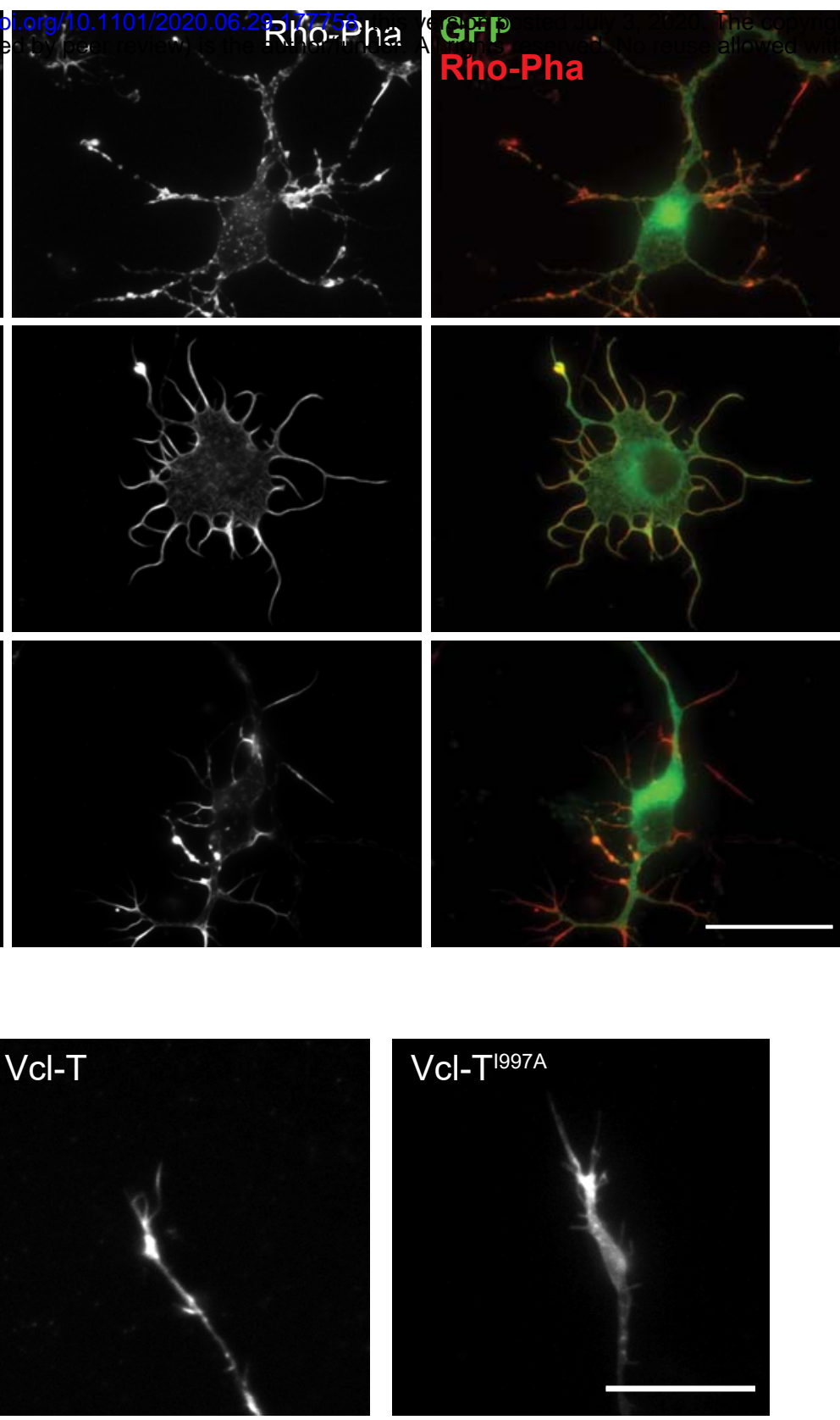

B

ht holder for this preprint (which ut permission.

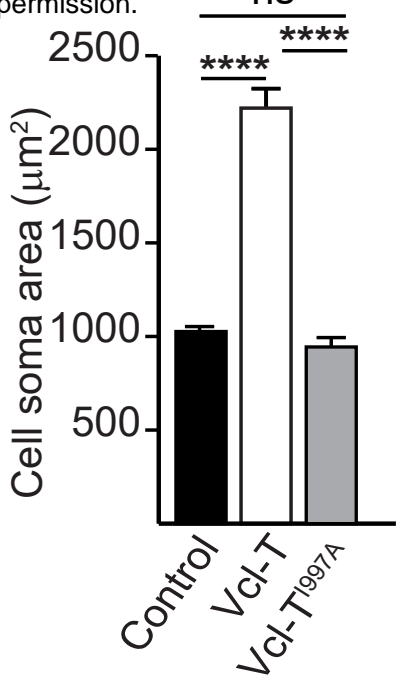

D

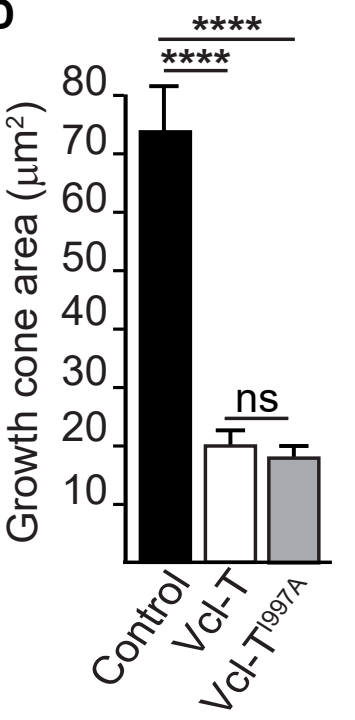


bioRxiv preprint doi: https://doi.org/10.1101/2020.06.29.177758; this version posted July 3, 2020. The copyright holder for thpplis preprint (which 6

A

\section{Control}

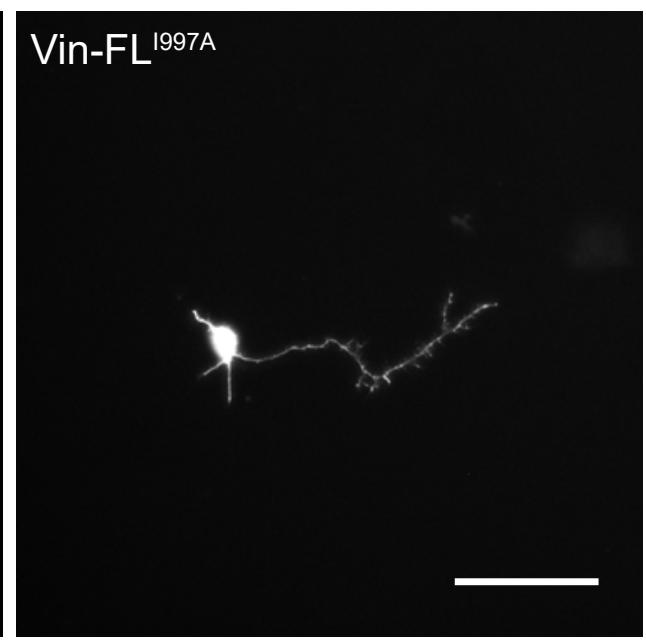

\section{B}
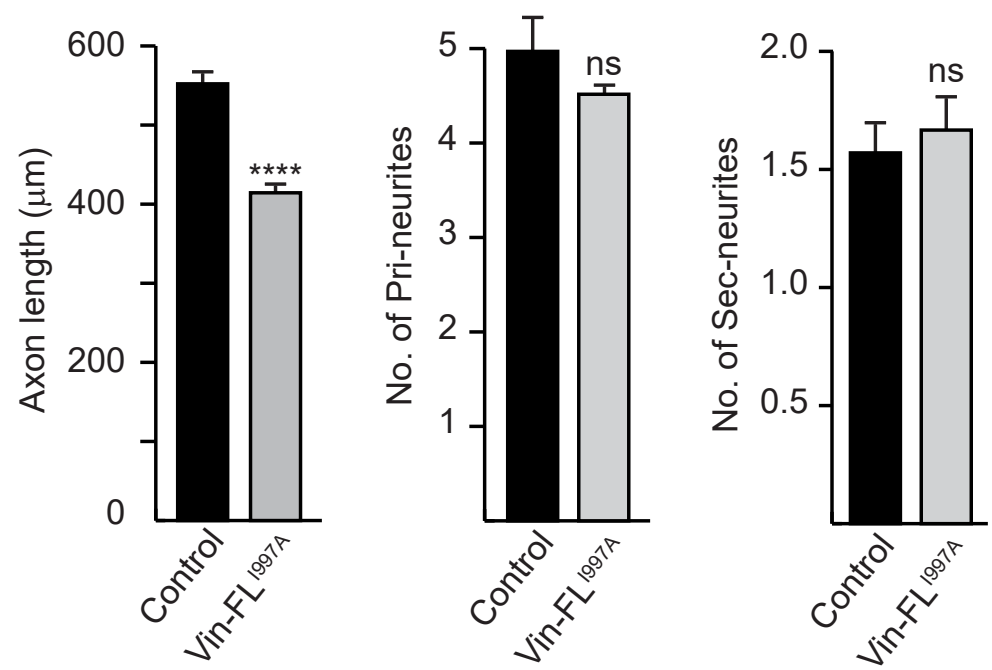
Suppl. Figure 7 bioRxiv preprint doi: https://doi.org/10.1101/2020.06.29.177758; this version posted July 3, 2020. The copyright holder for this preprint (which

A
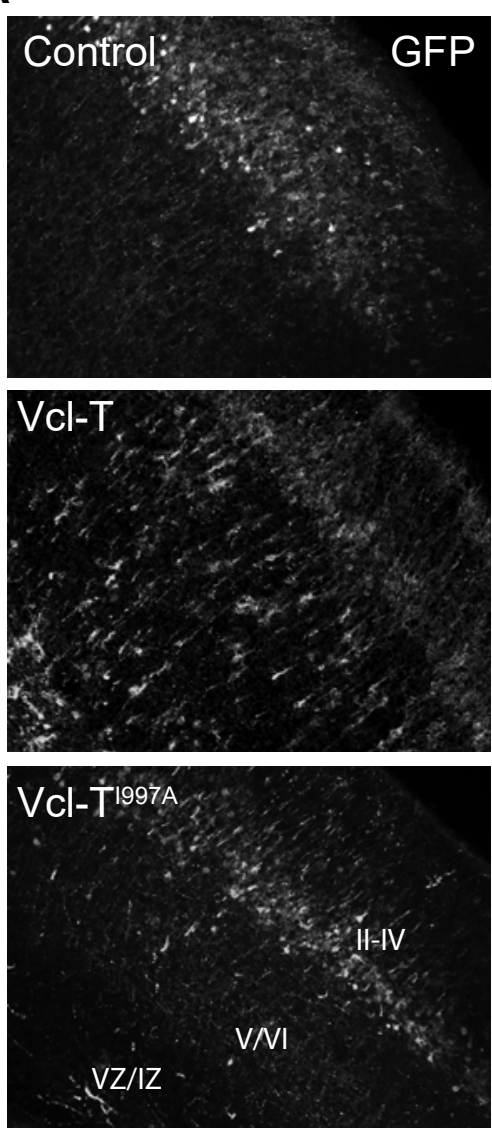
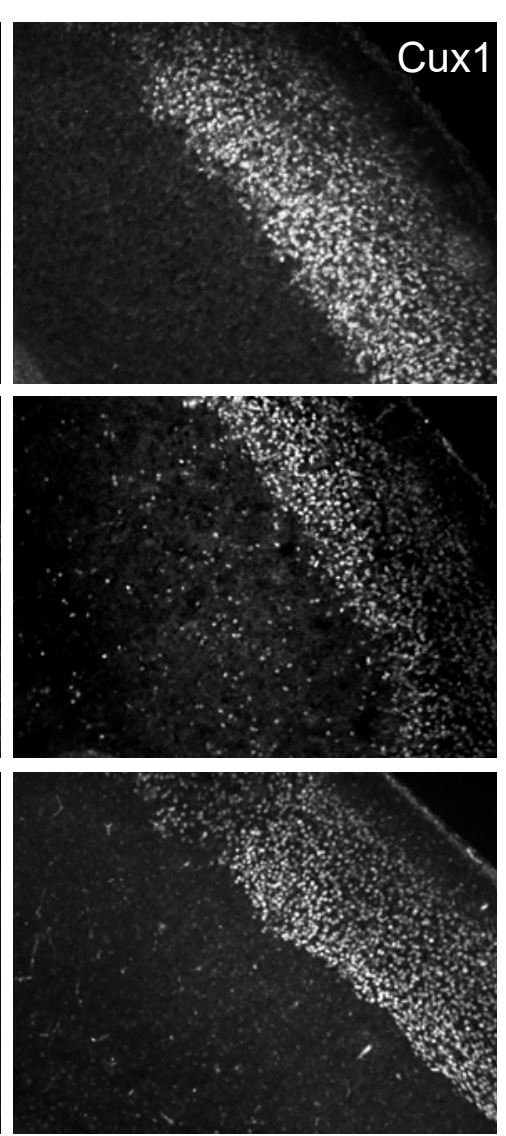
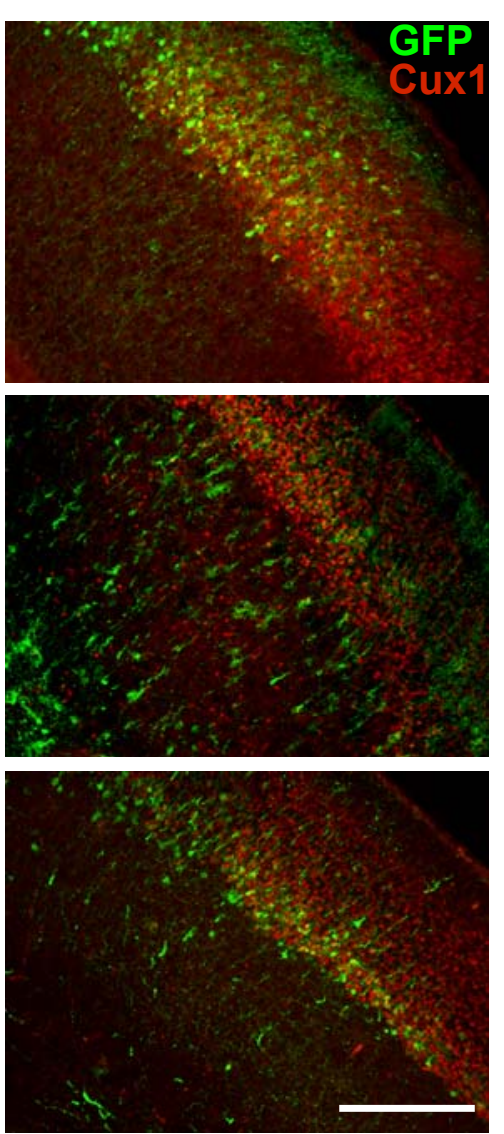

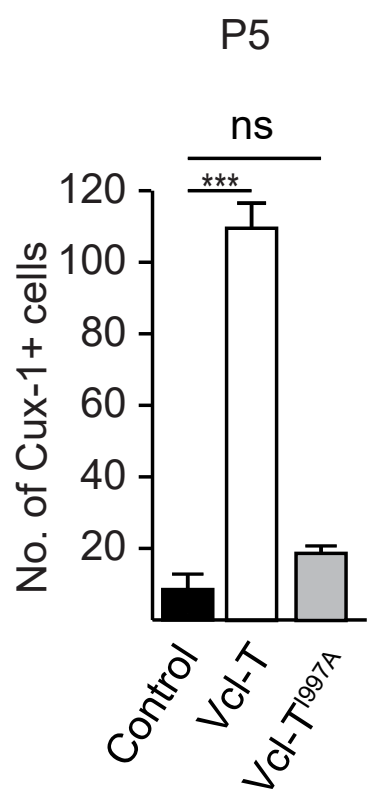

